\title{
Categorical framework for mathematical sense making in physics
}

\author{
Julian D. Gifford@* and Noah D. Finkelstein \\ Department of Physics, University of Colorado Boulder, Box 390, Boulder, Colorado 80309, USA
}

(Received 12 March 2020; accepted 25 August 2020; published 22 September 2020)

\begin{abstract}
We present a framework designed to help categorize various sense making moves, allowing for greater specificity in describing and understanding student reasoning and also in the development of curriculum to support this reasoning. The framework disaggregates between the mechanisms of student reasoning (the cognitive tool that they are employing) and what they are reasoning about (the object). Noting that either the tool or object could be mathematical or physical, the framework includes four basic sense making modes: Use of a mathematical tool to understand a mathematical object, use of a mathematical tool to understand a physical object, use of a physical tool to understand a mathematical object, and use of a physical tool to understand a physical object. We identify three fundamental processes by which these modes may be combined (translation, chaining, and coordination) and present a visual representation that captures both the individual reasoning modes and the processes by which they are combined. The utility of the framework as a tool for describing student reasoning is demonstrated through the analysis of two extended reasoning episodes. Finally, implications of this framework for curricular design are discussed.
\end{abstract}

DOI: 10.1103/PhysRevPhysEducRes.16.020121

\section{INTRODUCTION}

Mathematical problem solving is a critical component of the physical sciences, and so a topic of great focus in science learning. As such, there has been a substantial amount of work in physics problem solving [1-3], mathematical problem solving in physics [4-10], the coordination of mathematics with other representational forms [11-15], scientific sense making [16,17], and mathematical sense making in physics [18-22]. This large body of work has identified both student-centric problem solving strategies aimed at helping students engage with mathematical formalisms more like experts [1-3] and researcher-centric frameworks for considering student use of mathematics in physics $[4-8,12]$. This broad work spans from large scale descriptions of math use such as epistemic games $[9,10]$ and general problem-solving strategies $[1,4,5]$ to small scale descriptions of individual cognitive acts $[11,12,19,21]$. We provide a framework that bridges these disparate efforts and can be used as a resource both for describing student reasoning regarding mathematics in physics and for structuring the design of curricula to support this reasoning.

After a brief discussion of scientific sense making in general, and mathematical sense making in particular, we

\footnotetext{
*Julian.Gifford@colorado.edu
}

Published by the American Physical Society under the terms of the Creative Commons Attribution 4.0 International license. Further distribution of this work must maintain attribution to the author(s) and the published article's title, journal citation, and DOI. present the framework that draws from sociocultural theories of mediated cognition in order to operationalize some of the cognitive activities of mathematical sense making. This framework seeks to strike a middle ground that bridges the existing work, benefiting from the generalizability of larger-grained descriptions and the operational specificity of finer-grained approaches. The framework is useful for both researchers studying student reasoning and also for teachers and curriculum designers interested in helping students build connections between mathematical and physical explanations of physical phenomena.

To demonstrate the utility of this framework for describing student reasoning, we present two extended case study analyses of student reasoning about introductory quantum systems in sophomore-level college physics courses. In doing so, we also introduce a representational tool that supports the analysis of student reasoning and, we posit, can be used to scaffold the design of curricula to encourage engagement in similar reasoning.

The development of this framework is part of a broader investigation into student reasoning and curricular design. This present work addresses the following questions: (i) can we create a framework to describe the varied ways students engage in mathematical reasoning in the context of physics, (ii) what are the basic cognitive units of this framework, and (iii) how do these reasoning units link with one another into recognizable episodes of sense making? Additionally, we present a compact representational format for describing these larger reasoning episodes and consider curricular implications. 


\section{A. Scientific sense making}

In the physics education research (PER) and science education communities, significant attention has been given to the construct of "sense making." Though used frequently (appearing in 239 articles published in Science Education and 272 articles published in the Journal of Research in Science Teaching in the last five years alone), the term sense making is often not used consistently and can refer to a variety of activities that all generally align with the somewhat circular Oxford dictionary definition: "the action or process of making sense of or giving meaning to something" [23]. This idea of meaning making is sufficiently general to be applicable in almost any learning situation, and because of this generality lacks sufficient specificity to hold concrete meaning across any of them. As Odden and Russ argue "if every activity that is 'good' for science learning falls under the umbrella of sense making, then having a precise theoretical definition of it becomes superfluous" [17]. In an attempt to bring clarity to the term, a large body of sense making literature has been reviewed and synthesized by Odden and Russ, leading them to posit a more specific definition of sense making as the "process of building or revising an explanation in order to 'figure something out' - to resolve a gap or inconsistency in one's understanding" [17]. In addition to providing this broad definition, Odden and Russ group the varied uses of sense making in the science education literature into three categories, or "strands," in which sense making is conceptualized as (i) an epistemological stance towards science learning, (ii) a cognitive process, and (iii) a specific discourse practice.

Under the first strand, sense making is viewed as an epistemological frame for a given reasoning activity, where the individual (or group) holds a belief that the ultimate purpose of the activity is the construction of an explanation that leads to a greater understanding of a given phenomenon-not simply the creation of an answer. Within the second strand, sense making is viewed as a cognitive activity that leads either to the construction of new knowledge or novel connections between existing knowledge elements. Finally, sense making in the third sense is viewed as a particular form of communication, distinct from "argumentation" more generally, that focuses on (often collaboratively) constructing an explanation for the purpose of understanding rather than persuading. Though these three conceptualizations of sense making are structurally different, spanning grain sizes from individual acts of cognition to a mutually distributed framing of the activity, they share the common theme of constructing an explanation to "figure something out." While still broad, this provides a useful foundation for operationalizing cognitive sense making moves that can unpack the intuitive "know-it-when-you-see-it" moments of sense making.

\section{B. Mathematical sense making}

While situating the present work in this general space of sense making, we also consider the more specific topic of mathematical sense making (MSM). Recent work on MSM has, as with sense making in general, used a variety of definitions; considering MSM to be the coordination of conceptual understanding with the use and interpretation of algebraic symbols [19], blended processing where conceptual meaning is combined with mathematical formalisms during the mathematical processing stages of problem solving [20], and "coherence seeking" between mathematical formalisms and functional relationships in the world [18]. We see two common themes throughout these definitions: first, that they all specify cognitive processes, and so generally conceptualize MSM to fall within the second strand of Odden and Russ's categorization of sense making; and second, that they all call for a blending or coordination of mathematical formalisms and some form of conceptual understanding. As part of a broader investigation of student reasoning and curriculum development in quantum mechanics, we have developed a framework that operationalizes some of the cognitive processes involved in MSM and provides an operationalized organizational approach that links prior work.

In defining MSM as a cognitive process, we draw from Vygotsky's notion of mediated cognition as the basis for higher order cognitive processes. In mediated cognition, a mediator (an artifact or tool) is employed to aid in a subject's understanding of an object, and complements the individual's direct interaction with that object [24]. A common schematic of this cognitive structure is a triangle with the vertices subject, object, and tool, shown as the upper (solid) triangle in Fig. 1. The line connecting the subject and the object represents a direct engagement with (or understanding of) the object (e.g., developed through physical experimentation and embodied cognition [25])

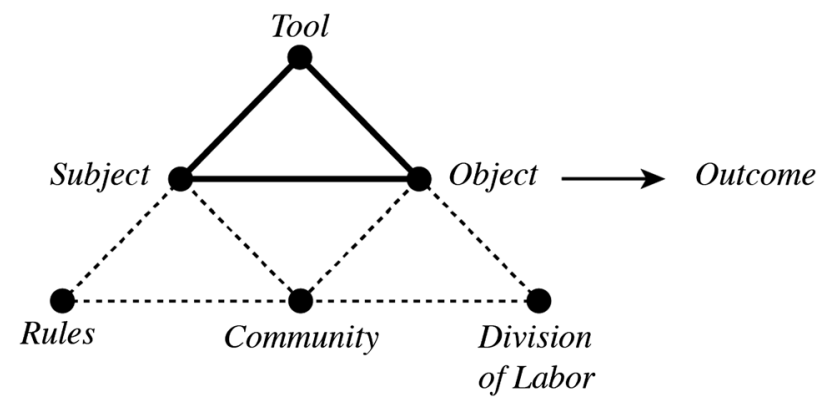

FIG. 1. A representation of the structure of activity, as described by activity theory. The upper (solid) triangle represents the mediated - by a tool-and direct pathways by which a subject interacts with an object, and is the unit of mediated cognition discussed by Vygotsky and others [24,26]. The other nodes in the triangle show the larger activity system, highlighting the connections between individual cognition and the larger sociocultural history in which the current activity is embedded. 
and the legs connecting the subject and object through a tool represent a mediated understanding of the object scaffolded by the use of the particular tool (e.g., using language, mathematics, or physical materials to mediate interaction with the object).

The larger triangle shown in Fig. 1 is a common representation of an activity system [27] (the fundamental unit of analysis of sociocultural systems), and provides an extension of Vygotsky's original work. Generally speaking, "activity" is the process by which objects are turned into outcomes. For example, a mathematical formula could be used to understand the behavior of a physical system. The activity of applying this formula (tool) to a particular physical system (object) in order to predict physical behavior (outcome) includes a cognitive process that is dependent on the roles and structure of the larger community, including cultural and historical rules for how tools are used and to what end. Thus, activity considers not only what object is used, but also how it is used and to what end it holds relevant meaning. Cultural historical activity theory suggests that activity "is the minimal unit that allows researchers to make sense of sense making by the research participants involved in the transformation of objects into outcomes" [28].

In other words, a complete description of the activity of sense making requires attending not only to the cognitive processes of an individual or group (the upper-most triangle) but also to the sociocultural environment in which the cognitive task is embedded. Such a framing aligns well with the general definition of sense making presented by Odden and Russ, where the outcome of the activity is an explanation and the three strands (epistemological frame, cognitive process, and collaborative discourse practice) speak to particular relations between the elements of the activity system. Mathematical sense making is then a subset of sense making activities that privilege the use of mathematical formalisms (as either tools or objects) in generating an explanation. In focusing on the cognitive process strand of mathematical sense making, both for an individual and a group, we attend primarily to the upper triangle of the activity system.

While activities of MSM privilege mathematical formalisms, reasoning that does not explicitly involve mathematics can contribute to a larger activity of MSM. Additionally, every instance involving formal mathematics does not necessarily count as mathematical sense making. However, to operationalize some of the cognitive processes involved in MSM it is useful to begin by considering the structure of mediated cognition, identifying both the objects and tools of sense making. Many of the existing frameworks that attend to the use of mathematics in physics [4-7] highlight either the mechanism or the object of the sense making but tend not to foreground both. Alternatively, some frameworks that do implicitly attend to both an object and a mechanism (or a process) do so on a grain size more comparable to activity [27,28], such as epistemic games $[9,10]$ or framing $[4,7,8]$. The framework presented in this paper builds on the existing models but attends to both the mechanism and the object of sense making at the grain size of cognitive moves described by mediated cognition. Distinguishing between these, while simultaneously attending to both, allows for a more nuanced understanding of student reasoning and provides greater specificity in considering the actions we wish students to engage in when designing curricula. Meanwhile, while existing frameworks that discuss student reasoning on a scale more like activity may overlap with what we will later call "molecules of sense making," the finer-grained approach of this framework can provide greater detail in describing the individual cognitive moves students make within these larger activities.

In discussing MSM we attend to the connections between mathematics and physics, but this requires definitions of what counts as "math" or "physics." Ultimately this distinction is somewhat arbitrary, and for many experts it may be neither clear nor productive. Indeed, the same activity may be seen as mathematical or physical in nature depending on the context and perspective. One productive framework, developed by Sayre and others [7,8], also defines four frames that include a distinction between math and physics without providing a specific definition of mathematics or physics. Rather, the authors rely on what seems to be a know-it-when-you-see-it heuristic to distinguish between the frames of algorithmic math and algorithmic physics, suggesting that algorithmic math is "performing mathematical computation by following well-established protocols without questioning the validity of those protocols," whereas algorithmic physics involves "recall[ing] physics equations or apply[ing] physics knowledge to rearrange known equations using math" [8].

While sensitive to the challenges of disaggregating these interwoven categories of math and physics sense making, we seek to provide analytical tools that allow us to explore learner engagement in MSM. To that end, and in line with existing work in PER, we provide a working definition and heuristic rather than a formal codified definition of entities as mathematical or physical. Our primary approach is to consider physics as emphasizing conceptual relationships and principles in the world and mathematics to be focused primarily on the use and manipulation of symbolic and graphical representations in a self-consistent fashion. A major distinction here is that mathematical objects or tools are not bounded by considerations of the physical system. This is in agreement with the characterization above that (algorithmic) mathematics involves performing computations using existing protocols. To clarify, we do not consider mathematics to be solely symbolic in nature; multiple representations (plots, graphs, etc.) can be mathematical, provided they illustrate a specific relationship between abstract quantities. While physics also deals with 
relationships between quantities, these quantities and relationships (and representations thereof) are anchored in the real world-or specific models of the world. Finally, in analyzing student reasoning we do not classify entities based solely on our beliefs as researchers, but on how the entity is discussed by the learner. This follows Dewey, who notes "the teacher should be occupied not with subject matter in itself but in its interaction with the pupils' present needs and capacities" [29].

\section{THE FRAMEWORK}

\section{A. Four modes of sense making}

Applying the notion of mediated cognition leads us to consider instances of scientific sense making where mathematics serves as either (i) the tool and/or (ii) the object of the cognitive process. Distinguishing these entities as either physical or mathematical leads to four general reasoning structures, or sense making modes: mathematical sense making of a mathematical object (Msm-M), mathematical sense making of a physical object (Msm-P), physical sense making of a mathematical object (Psm-M), and physical sense making of a physical object (Psm-P). The four modes are shown in Fig. 2 and an example of each, present in the existing literature, is discussed below.

Psm-P: Much of the early work in PER focused on determining and cataloging common student difficulties with the concepts of introductory physics. This basic research was then used as a base for the development of curricula. These research-based curricula generally aimed to develop conceptual reasoning skills to help students build physical models (often excluding formal mathematics) to describe physical phenomena. A canonical example comes from the University of Washington's study of

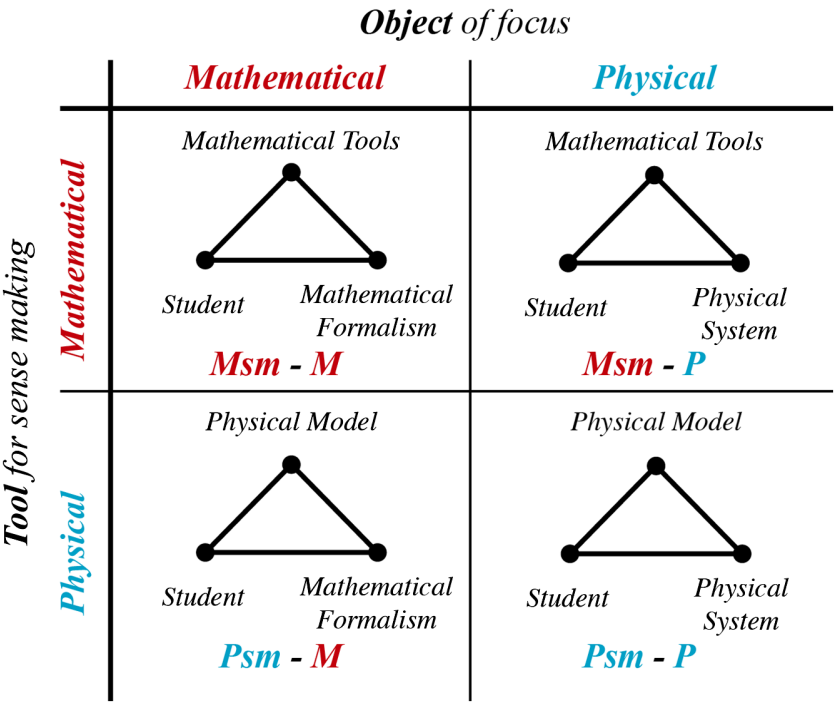

FIG. 2. The four reasoning structures (modes) of the categorical sense making framework. These modes differ based on the object of the sense making and the tool employed in the sense making. student reasoning regarding electric circuits [30,31]. The curricula developed out of these studies [32,33] scaffold students in building a conceptual, qualitative model of electric circuits, encouraging the use of physical reasoning as a tool for understanding a physical system.

As discussed by Shaffer and McDermott [31], this conceptual model is built up through physical experimentation and begins with the single concept of electric current. Students come to understand that current will split or recombine when it encounters a junction, but that it is not used up. Using this physical, qualitative model they are able to accurately rank the brightness of the configuration of bulbs shown in the left of Fig. 3 by arguing that all of the current leaving the battery passes through bulb A, splits equally at the junction since the two branches are identical and so bulbs $\mathrm{B}$ and $\mathrm{C}$ are equally bright but about half as bright as $\mathrm{A}$, and then recombines such that all of the current passes through bulb $\mathrm{D}$ making it the same brightness as $\mathrm{A}$ $(\mathrm{A}=\mathrm{D}>\mathrm{B}=\mathrm{C})$. While there is some number sense included in this explanation (that the current splits equally) the explanation is not formally quantitative as bulb brightness is an approximate, nonlinear measure of current. Additionally, as shown in the right of Fig. 3, often questions in the University of Washington's curriculum explicitly request that students not rely on mathematical formalisms in their explanations. This is not to say that physical reasoning cannot be quantitatively accurate, or that the presence of numbers necessitates categorization as mathematical reasoning. Consider, for example, that there were a third parallel branch in the circuit described above. Arguing that the current would split evenly, and so each of the parallel bulbs would be a third as bright as bulb A, is quantitatively accurate (within the scope of a model of brightness as a roughly linear indicator of current) while still primarily representing the use of a conceptual physical model as a tool.

By encouraging conceptual modeling, and sometimes providing explicit cues excluding mathematical reasoning, these curricula encourage students to engage in a Psm-P mode of reasoning. While this mode in isolation is not an example of mathematical sense making, the coordination of Psm-P with the other modes can contribute to a larger
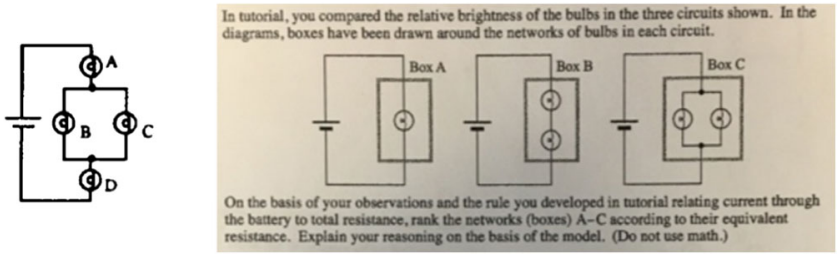

FIG. 3. Left: a circuit diagram used as an evaluation of the conceptual circuit model developed by Shaffer and McDermott [31]. Right: a homework question from the Tutorials in Introductory Physics. Students are asked to rank the resistances of the boxed circuit elements and are explicitly told to not use math in their explanations. [33]. 
activity of MSM - as will be explored in the case studies below. This example supports a major goal of this framework: having operationalized these sense making modes, both authentic student reasoning and curricula can be analyzed by considering the reasoning structures (and the connections between them) that are present in learner reasoning or scaffolded by the curriculum.

Msm-M: Perhaps the most "mathematical" mode would be using mathematical reasoning as a tool for understanding a mathematical object (Msm-M). In this mode mathematical reasoning is utilized to understand an object that is mathematical in nature (an equation, graph, etc.) and does not focus on any explicit connection to the physical system. This is not to suggest that the mathematical object does not have physical significance, rather that in an Msm-M mode these physical properties are not the focus of attention.

Work by Hahn et al. [19] studied physics sense making strategies in a middle-division classical mechanics course, largely focused on evaluative answer-checking types of reasoning. In class and on homework, after obtaining a mathematical answer, students were explicitly asked to consider whether the result made sense using specific evaluative strategies such as unit analysis and special-case analysis. In special-case analysis the expression is evaluated at a specific value that generally corresponds to recognizable physical behavior, however whether or not these special cases prove physically relevant for sense making depends on the individual students. While these techniques offer an opportunity for students to connect their mathematical result to the physical characteristics of a particular system (encouraging Msm-P and/or Psm-M, see below), this physical connection is not always explicitly scaffolded as both the tool and object are generally mathematical. In particular, we are not arguing that special-case analysis never includes a physical tool or object, only that the solution presented below focuses on the use of mathematical protocols to eventually relate one mathematical equation to another, mostly brushing over the process of mapping physical characteristics to symbolic values.

In her analysis of student sense making Hahn examined responses to an explicit special-case analysis prompt. The multistep homework problem walks students through an analysis of projectile motion on a flat but not horizontal surface, a diagram of the problem setup is shown on the left of Fig. 4. Part (e) of the problem asks students to determine an expression for the maximum range $R$ of a projectile launched with speed $v_{0}$ at an angle $\theta$ above the ground when the ground makes an angle $\phi$ to the horizontal, which gives the expression $R_{\max }=v_{0}^{2} / g[1+\sin \phi]$. Part (f) explicitly asks students to use special-case analysis for $\phi=0$ and $\phi=\pi / 2$ to determine whether or not their expression makes sense. The solution provided by the TA, shown on the right of Fig. 4, evaluates the expression for the two cases and relates them to two previously

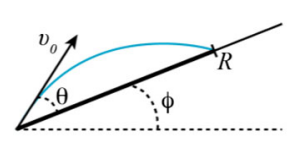

f) Sense-Making: Consider Special Cases Does your result for the maximum range make sense for if the ground is horizontal? If the ground is vertical (like right up Solution: If the plane is parallel to the ground, $\phi=0$ and the expression becomes
$\max =\frac{v_{g}^{\prime}}{g}$, which matches the result from homework 1 . If vertical, $\delta=\pi / 2$ and it becomes $_{\max }=\frac{v_{2}^{2}}{2 g}$, now corresponding to the maximum height that we also found on homework 1 (height and distance along the ramp now being equivalent)

FIG. 4. Left: a diagram of the angled-ground projectile problem, drawn here for clarity. Right: the solutions to part f) of the angled-ground projectile problem, as presented by Hahn [19].

determined formulas, the max height and max range of a projectile on level ground. We classify the reasoning presented in this solution to be Msm-M as the object (the expression for the maximum range) is mathematical and the tool (special case analysis and the resulting expressions) is also mathematical. Through the use of this mathematical tool, the TA is able to determine that the derived expression for max range is reasonable as it simplifies to other well-known mathematical relationships. By categorizing this reasoning as Msm-M we do not mean to suggest that this solution is incomplete, or that it does not represent physics sense making. Rather, we highlight this as an example of productive sense making in the context of physics where both the tool and object are mathematical. In contrast to other definitions of MSM that require a blending of mathematical formulas with conceptual reasoning regarding the physical world, this framework values this "math only" reasoning as a potentially valuable form of MSM.

Msm-P: Perhaps the most canonical view of MSM would be instances when a mathematical formalism is leveraged to understand the behavior of a physical system. In practice, this is often a complicated process, perhaps requiring Msm-M type reasoning to understand the formalism before it can be used to understand physical behavior. Despite this complicated structure, it is still possible to identify local instances of Msm-P, such as in Kuo et al.'s analysis of a mathematical "shortcut" in which a student leverages their understanding of the expression $v_{f}=$ $v_{0}+a t$ as describing a "base plus change" process to determine the difference between the final velocities of two balls thrown with different initial velocities [21]. In their study, Kuo et al. contrast two particular solutions methods, claiming that one shows opportunistic blending of physical and mathematical reasoning (their definition of MSM) while the other is a more traditional plug-and-chug solution.

In the plug-and-chug solution, as shown in Fig. 5, the student inserts the given values into the velocity equation to solve for the final velocities of each of the balls and then takes the difference between them. When asked explicitly if there was a way to answer this problem without calculating the answer, this student says it should be possible but doesn't know how to do so. This solution might be considered to be Msm-M, where a (mathematical) formula is used as a quantitative tool to solve for the requested (mathematical) quantity. If the student went on to explain 


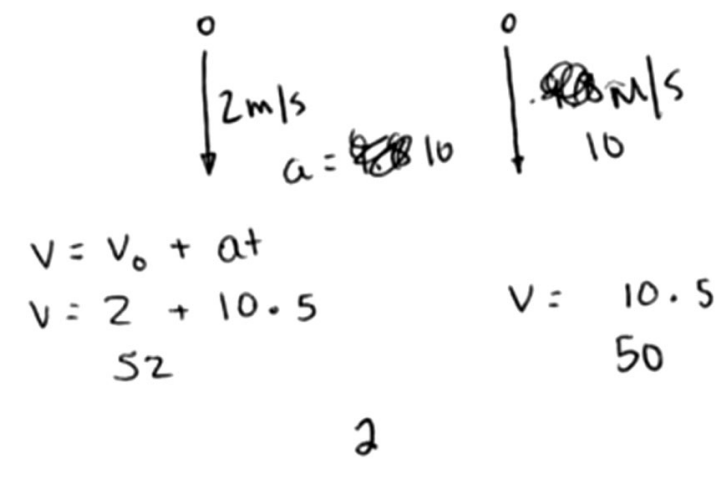

FIG. 5. Student work from Kuo et al. [21] exemplifying a "plug and chug" type of solution.

what their calculated result means about the physical motion, we would consider this a useful step in a larger activity of MSM - again arguing that math only work can be a useful aspect of sense making. However, because this student does not continue on to make further connections, we argue that this does not meet the larger criteria of sense making (constructing an explanation that leads to greater understanding) and so is not an instance of MSM.

Kuo et al. contrast this solution with reasoning they feel exhibits greater MSM. In the second approach, after writing the symbolic expression for the velocities, the student uses the conceptual argument that "...the acceleration is a constant and that means that velocity is linearly related to time... so the first differences [the slopes] are the same, and if the first differences are the same then the initial difference between the two speeds should not change." Kuo et al. note that this reasoning allows for a shortcut, where the student is able to make use of the equation to understand the motion without explicitly calculating values. We agree that this reasoning is MSM, specifically Msm-P where the equation is used as a (mathematical) tool to understand the (physical) motion of these two balls. One might argue that this example shows multiple reasoning modes, where the student engages first in Msm-M to interpret the equation as describing a base plus change process and then Msm-P (using the interpretation of the equation as a scaffold to consider the changes in velocity of the balls). Ultimately, this is a question of timescale, and we will address the subsequent use of multiple modes further below.

Psm-M: In the final mode of the framework, physical reasoning is used as a way to understand a mathematical object. As with Msm-P, this mixed reasoning mode is likely done in conjunction with another mode (e.g., engaging in Psm-P in order to leverage a conceptual understanding of the system and then applying this as a tool to understand the mathematical formalism). However, it is also possible to observe local instances of Psm-M, for example, OSU's use of three-dimensional surfaces as a way to understand and conceptualize partial derivatives [34]. These surfaces can provide a physical scaffold that explicitly shows how partial derivatives (slopes of the surface) depend on the direction of change.

There is no explicit hierarchy to these modes, i.e., engagement in any one mode is not fundamentally better or more important than engagement in another. When considering sense making of physical and mathematical phenomenon, each mode is a potentially productive reasoning structure. While in specific instances a particular mode may be preferred, expert reasoning likely involves fluidity with all of them and an understanding of which mode may be most productive in a given situation. For example, the two mixed reasoning modes (Psm-M and Msm-P) are likely at play during instances of what Kuo et al. have called "calculation-concept crossover" [22] where conceptual reasoning is used to answer a quantitative question or calculations are used to answer a qualitative question.

The framework provides tools to discuss both student reasoning (what tool are students using) and metareasoning (do they feel it is the right tool to be using). Part of the utility of this framework is that it provides a language for discussing the small-scale actions that students engage in. However, the framework does not attempt to fundamentally redefine sense making, and we do not claim that these four modes are a complete description of all possible sense making. Rather, the modes of this framework offer a way to operationalize the moves students engage in that might contribute to a broader activity of MSM.

\section{B. Shifts and connections between modes}

In applying the framework to student reasoning, we use the four modes of the framework as basic building blocks in our analysis. However, we note that these modes are of a middle grain size. They specify a cognitive activity that is larger than the activation of a cognitive resource [35] or the use of a phenomenological primitive [36] but are generally smaller than the larger activity of sense making, epistemic games, or framing. These modes can be viewed as "atoms" of reasoning, and while it is possible to identify instances where engagement in a single mode shows robust sense making, often it is the combination of these modes into "molecules" that aligns with the larger activity of mathematical sense making. In addition, as discussed above, these atoms and molecules of sense making are embedded in sociocultural systems that include epistemological framing, individual and collective history, and metacognitive reflection. Though we believe all of these to be contributing factors to the larger activity of sense making, we limit our focus to learner engagement in these reasoning modes and the processes by which these atoms of reasoning are combined into molecules of sense making. Here, we present three processes by which the modes are combined, translation, chaining, and coordination. We do not claim that this is an exhaustive list, only that these three processes are prevalent in the data we have analyzed and productive in describing student reasoning. 


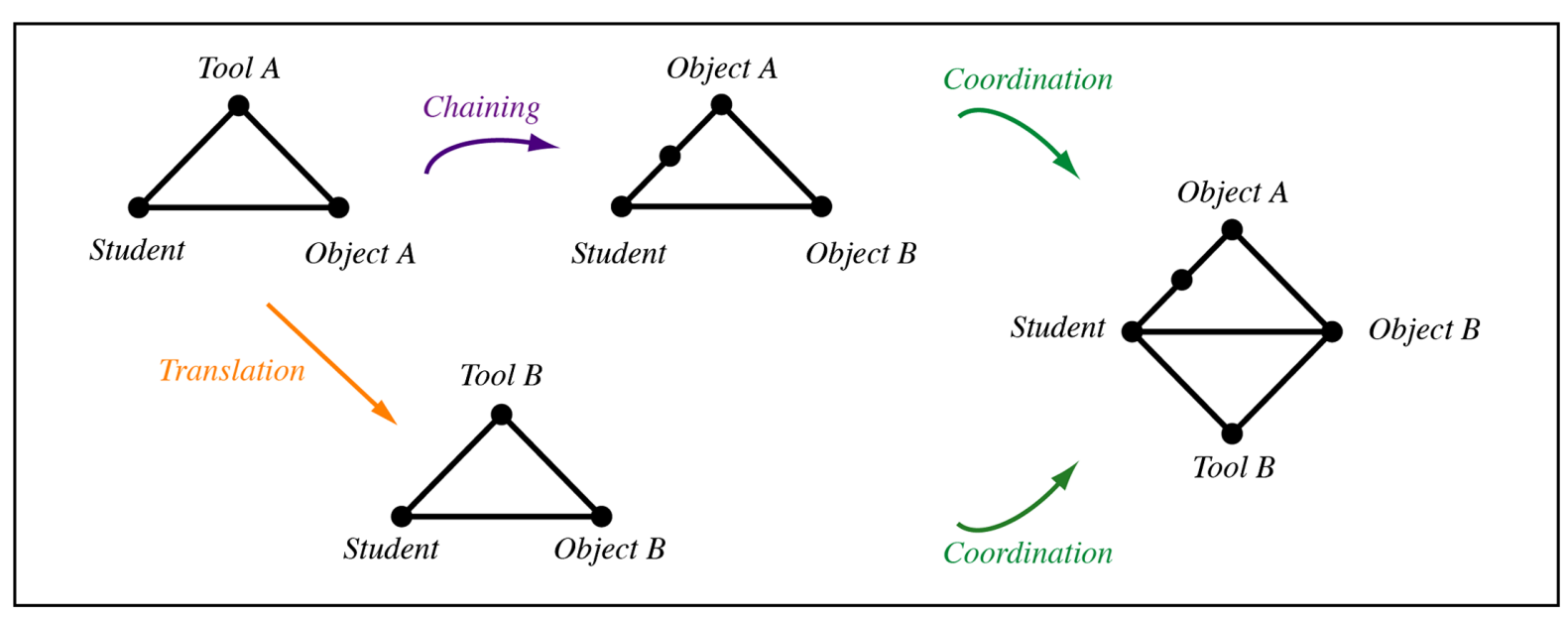

FIG. 6. An example of a sense making diagram. These diagrams show the individual atoms of reasoning as well as the processes by which they come together to form a molecule of sense making. Each individual shape (triangle, triangle with node, square, etc.) represents a unique reasoning structure, which can be either a local instance of a single mode, or be built up of many modes through the processes of translation, chaining, and coordination.

The simplest change in reasoning structure is a translation from one of the four modes to another. Since the modes are defined based on the tool and object of sense making, a translation occurs whenever the tool and/or object of focus has changed. In the sense making diagrams below translation appears as a straight (orange) arrow that connects one reasoning mode to another, as shown in Fig. 6. In this abstract example, both the tool and object have changed from the first mode to the second. Translation here is akin to Bajracharya et al.'s process of representational translation [11] in that it describes a shift in categorical structure; however, translating between reasoning modes defined by tools and objects and translating between representations are likely different cognitive processes.

Though a translation does not require there be a specific connection between the two modes, it is often the case that there is a link. As suggested in the discussion of the two ball problems above, there are many instances where prior reasoning builds to create a chain of reasoning modes. In creating this chain of modes, it is common for the object of reasoning in one mode to become the tool used in the next mode. This special translation between modes is called chaining. The process of chaining is represented as a curvy (purple) arrow that creates a node between the subject and the tool, as shown in Fig. 6. This node represents the use of tool $\mathrm{A}$ as a mediator in understanding object $\mathrm{A}$, which has now become the tool used to understand object B. In these sense making diagrams the vertical "axis" represents different reasoning structures (usually represented as triangles), while the horizontal axis shows the progression of time.

Another common link between modes occurs when the object remains the same but different tools are used in the MSM process. This form of connection represents students using two different tools to mediate their understanding of the same object. Coordination is the process of combining these two different approaches. Bajrachara et al. discuss the process of representational consolidation [11], through which information from several different representations is combined into a single representation; this is likely a specific form of coordination. Coordination is represented by two curvy (green) arrows that combine the two previous triangles into a coordinated structure, as shown in Fig. 6. To make the coordinated structure, one of the previous triangles is flipped, noting this view is for visual clarity only. There is no preference for which triangle is flipped, though generally it is the reasoning structure that was used second as it appears lower on the diagram. A detailed example of this process is provided in the case studies below.

To help track the assembly of these reasoning modes into a molecule of sense making we present a flowchartlike visual representation, as shown in Fig. 6, called a sense making diagram. These diagrams are akin to Podolefsky's analogical scaffolding diagrams [37] in that the focus is on the formation of complex reasoning structures; but the specific attention to the processes of translation, chaining, and coordination give them a similar thematic feel to representational transformation diagrams of Bajracharya et al. [11]. Though the framework presented here was developed independently from the work of Bajracharya et al., we see the many analogies between these frameworks as mutual support for the efficacy of these approaches. Additionally, we argue that sense making diagrams can be used as an analysis tool for MSM in a similar fashion to the use of RT diagrams in considering multirepresentational problem solving.

In the present work we have largely employed this framework to analyze think aloud reasoning that shows inthe-moment construction of a molecule of sense making. This rich dataset provides a detailed view of learner reasoning on a problem they have not necessarily encountered before, where it is possible to identify instances of 
chaining and coordination as learners build up their understanding. However, it is likely that once these novel connections are made learners will be able to apply their more complicated reasoning structures without explicitly going through the steps of chaining and coordination again. This process, by which complicated reasoning structures built through chaining and coordination collapse into an immediately applicable reasoning structure, can be considered compilation. In his work on analogical scaffolding [37], Podolefsky suggests that expert reasoning can be described in terms of these compiled structures, and that experts are likely not always conscious of the individual ideas that have been compiled.

\section{METHODS}

In what follows, this framework is applied to analyze two separate reasoning episodes in which students work collaboratively on an extended problem. The data for each of these reasoning episodes come from one-time, end of semester focus groups. Both focus group interviews were conducted by the first author, they were audio and video recorded, and student work (both individual and collective) was collected and scanned. The focus groups were held in different semesters, with students from different modern physics classes, and the interview protocols (the questions asked) differed in content. In the analysis of these different situations we show both the utility of the framework in describing student reasoning and also suggest its general applicability beyond a particular course or content domain.

The data for the first case study come from students enrolled in a middle-division modern physics course at CU primarily intended for engineering majors (largely mechanical and electrical). The participants are three students from this course, with pseudonyms Amanda (white, she/her), Ben (white, he/him), and Jack (white, he/him). The second author was the professor of record and the first author played a substantial role in both curricular development and in-class implementation. Though collaborative group work is a major emphasis in this classreinforced through ConcepTests (clicker questions) [38], Tutorials [33], and group exams-these students had not worked together consistently before the focus group. Participation in the focus group had no direct effect on these students' course grades. Students volunteered in response to an email sent to the entire class, and were compensated monetarily for their participation in the 90-min focus group. While some effort was made to ensure that the students were somewhat representative of the class (i.e., selecting students with varied course grades) and that there was not a group with three men and one woman, the choice in students was primarily based on scheduling availability. The protocol was written by the authors, drawing on content domains previously highlighted in the course. Background on the particular prompt is discussed in the case study I section below. While none of the questions were directly pulled from class, and so all questions were novel for these students, analogous content had been covered both in class and on the homework.

Since the lecture instruction and curricular materials for this course were developed by the authors, with our view of mathematical sense making in mind, it is reasonable to consider our engagement a confounding factor in these students' reasoning. To address this, we include a second case study with students from a course in which we played no direct role in instruction or curricular design.

The data for this second case study analysis come from another one-time, end of semester focus group from a middle-division modern physics course at $\mathrm{CU}$ Boulder. However, while the course taken by the students in the first focus group is primarily intended for engineers, this course is primarily intended for physics majors. While much of the content is similar between the two courses-both are a general introduction to quantum mechanics, specifically discussing the Schrödinger equation and simple 1D systems including square wells and tunneling - the majors' course tends to be more traditional in its instruction and relies more heavily on mathematical calculations. The first author was the interviewer, but outside of this focus group neither author was involved in this particular course. Students were recruited through an email to the entire class and were paid for their participation in the $60 \mathrm{~min}$ focus group. Four students were recruited, Penny (white, she/her), Morgan (white, they/them), Cam (white, he/his), and Sarah (white, she/her). Sarah speaks very little over the course of the focus group, so the collective reasoning is mostly done by Penny, Morgan, and Cam. The majority of the written work in the session was produced by Morgan. The social dynamics of this second group are complex, and we discuss the interplay between social dynamics, reasoning, and sense making in another paper [39].

The interview protocol for the second focus group was substantially different from the first. As the authors were not involved in the course, we had little sense of what topics were presented. Thus, the protocol was decided based on the topics listed in the course syllabus and a general desire to see students engage with questions that required multiple representations (symbolic, graphical, etc.). The protocol was finalized through discussions with members of both the PER group at $\mathrm{CU}$ and in collaboration with members of the PER group at the University of Maryland.

The analysis process was identical for both focus groups: they were audio and video recorded and artifacts (written work, both individual and collective) was collected and scanned. The first author watched the recordings repeatedly, breaking them into reasoning episodes based on changes in content, the object of focus, or the approach to problem solving-particular attention was given to instances where mathematical formalisms played a key role. Reasoning episodes that were flagged as representing sense making in an intuitive know-it-when-you-see-it way 

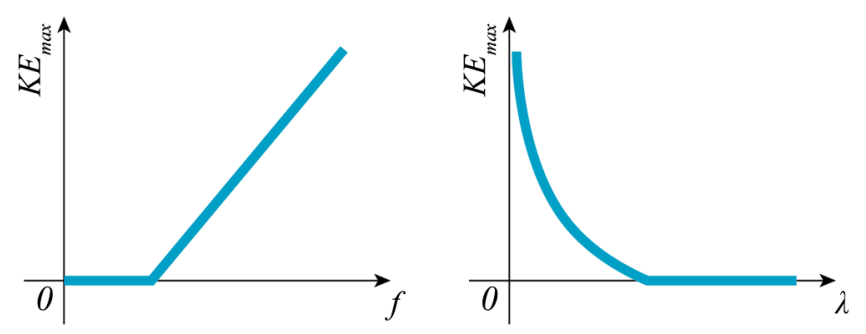

FIG. 7. Qualitatively accurate plots of KE vs $f$ (left) and KE vs $\lambda$ (right).

were transcribed by the first author who then conducted a preliminary analysis.

The flagged reasoning episodes and the accompanying transcript (but not the preliminary analysis) were shared with the second author. After a brief collective discussion of the reasoning episode, including reaching agreement that the reasoning episode showed intuitive sense making, the preliminary analysis was presented and discussed. During this discussion the authors reached agreement on the coding of reasoning modes and the development of the molecules of sense making. These joint analyses were presented at multiple PER group meetings at CU and in collaborative research meetings with members of the PER group at the University of Maryland. The analyses were refined based on feedback from these presentations, and the final description of student reasoning presented here was mutually agreed upon by both authors. While we did not engage in a formal IRR process, the analyses have been agreed upon by the authors, incorporating feedback from the multitude of group meetings.

\section{CASE STUDY I: AMANDA, BEN, AND JACK}

This first case study is an extension of a brief, initial analysis used to demonstrate proof of concept of the utility of the framework [40]. Here, we expand on the previous analysis, drawing from a larger section of the focus group interview and highlighting how the students' activity of sense making can be described in terms of the development of a complex "molecular" reasoning structure through translation, chaining, and coordination of the individual sense making modes. This fine-grained analysis shows how the framework can be productively applied to describe and understand student reasoning, and how this reasoning can be represented in the form of sense making diagrams.

\section{A. Context and prompt}

A major content focus in the first third of the course is the development of a holistic model for light. Beginning with a model of light as an electromagnetic wave, students consider the wavelike behavior of light including interference, intensity of a sustained beam, and the relationship between frequency and wavelength. Students then apply this model to the photoelectric effect, and the experimental observations lead them to develop a quantum (photon) model of light. An important piece of experimental evidence supporting the photon model is the existence of a "cutoff frequency," the minimum frequency of light that will eject electrons from the surface of a material. Above this frequency, the energy of the ejected electrons increases linearly, suggesting that the energy of the light depends directly on the frequency - a plot of the maximum kinetic energy of the ejected electrons versus the frequency of light is shown in the left of Fig. 7. While this dependence on frequency is not described by the wave model, it does indicate that the behavior of the photons is related to properties of the wave, suggesting an overlap between these two models of light.

At multiple points throughout the course - a pretest (week 1), a homework (week 3), and a free response exam question (week 5) - the students were asked to consider plots and functions representing the maximum kinetic energy of the ejected electrons versus the wavelength of incident light. While the plot of KE versus frequency is linear, $\mathrm{KE}$ is inversely proportional to wavelength. Because of the inverse relationship between frequency and wavelength, the minimum cutoff frequency is equivalent to a maximum cutoff wavelength, above which the KE is zero as no electrons are ejected from the plate. A qualitatively accurate plot of KE versus $\lambda$ is shown in the right of Fig. 7 . Throughout the semester, students were shown a piecewise functional form for the KE as a function of the frequency, initially with the physics context removed:

$$
f(x)= \begin{cases}0, & \text { for } x<\frac{B}{A} \\ A x-B, & \text { for } x \geq \frac{B}{A}\end{cases}
$$

Given the relationship $x=D / y$, students were asked to determine a plot of $f(y)$ and relate this mathematical formalism to the photoelectric effect, interpreting $f(y)$ as the function $K E(\lambda)$. On the exam, students were asked explicitly to sketch a plot of KE versus $\lambda$ without the formal symbolic scaffolding. In the end of semester focus group, from which the following data are taken, these students were asked to consider two photoelectric effect experiments in which identical light is incident on plates of different work functions $(\Phi)$ - a measure of how energetically bound the electrons are, which determines the minimum photon energy required to eject electrons from the plate. The students were asked to make plots of KE vs $\lambda$ for these two experiments; their work, drawn by Amanda, is shown in Fig. 8.

In the analysis below we distinguish three distinct but complimentary "scenes" in this reasoning episode. The analysis that follows is conducted separately across these three scenes. Each section includes transcripts of student reasoning followed by our analysis of that reasoning and its relation to the previous stages of their work. In the 

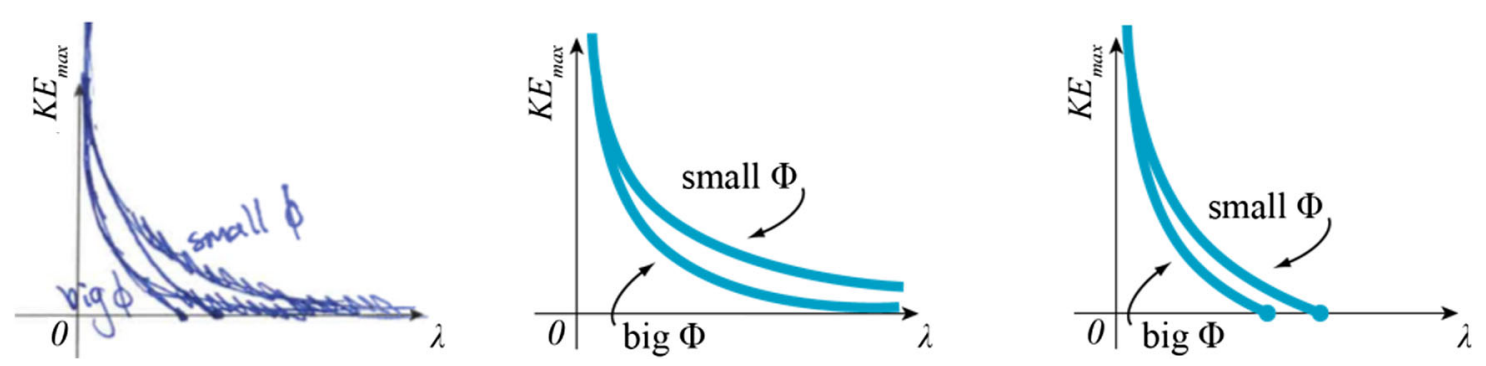

FIG. 8. Student work by Amanda. The leftmost picture is a scan of her actual work, showing her initial answer (the crossed-out plots) and her final answer. For clarity, we have included plots separating her initial sketch (center) and the group's final answer (right).

transcripts, ellipses represent omissions in the complete student dialogue made for clarity and length.

\section{B. Scene I: Sketching initial plots}

A: I was gonna draw something sorta like, uhhh, that [center plot in Fig. 8]. 'Cause uhh the shorter the wavelength is the more powerful the light is so the more energy this [the electron] has when it bounces, when it comes off of the plate.

$J$ : And then the other one with the different uhh work function would be always less than that? Or, because wouldn't it [the electron from plate 2] always have less kinetic energy because it leaves with less?

A: Well, I think it would still have the same like asymptotes here $[\lambda=0]$... but like it would dip further down closer to this [the $\lambda$ axis].

$J$ : And remain under it [the plot for plate 1] the whole time.

A: Yeah... I agree with you.

In this exchange Amanda and Jack are attempting to generate a graph of $\mathrm{KE}$ vs $\lambda$. While this graph inarguably has physical significance, in this moment the students treat this plot as a fundamentally mathematical object, expressing a formal relationship between two variables. However, Amanda's reasoning is physical in nature (that shorter wavelength light is more powerful and will cause electrons to leave with more energy). We take this as evidence that Amanda is engaged in a Psm-M mode of reasoning. Jack also joins in this mode, suggesting that the second plot be "always less than" the first because electrons are ejected from the second plate with less energy (for the same wavelength). While one might argue that Amanda's use of the inverse relationship between wavelength and energy is primarily mathematical reasoning, her use of the phrase "more powerful" suggests that she is reasoning about relationships between physical quantities, rather than purely mathematical variables. As this is the beginning of the reasoning episode, it is perhaps not surprising that the students are engaged primarily in one reasoning mode (Psm-M). There is a single object of attention (the plot of $\mathrm{KE}$ vs $\lambda$ ) and they have collectively been using a single tool (physical argumentation regarding the "power" of the light). This reasoning mode is diagramed in scene I of Fig. 9. Note that all three scenes of this reasoning episode are contained in this single figure. These students go on to use their plot, currently the object, as a tool in future reasoning, so the figure shows a "collapsed" representation of the diagram. In the collapsed diagram, the node between the students and the plot represents the use of physical reasoning as a tool in understanding the mathematical plots.

At this point, the students have agreed that the plots should be inverse decays (they have drawn the incorrect plot in the center of Fig. 8) but have not indicated the cutoff wavelengths or their relation to the work function. Additionally, while Amanda has qualitatively related the wavelength to how "powerful" the light is, the students have not used any explicit mathematical formalisms to scaffold their reasoning. Despite this, the students feel they have answered the question fully (Amanda saying "well that's this round of questions") at which point the interviewer asks them if there are any relevant mathematical equations that might model the graphs they have drawn. The students agree that there are, but that they do not remember them. In the next scene, the group shifts their focus to a new object: a symbolic representation (equation) for $K E(\lambda)$.

\section{Scene II: Determining the equation}

After a period of "equation hunting" through their notes, Ben suggests

$\boldsymbol{B}:$ These [the plots] are definitely inverse relationships. What about, possibly, it seems to follow the relationship of KEmax over $\lambda$. As $\lambda$ increases this [the graph] decreases like a curve.

A: ...You're right about the $\lambda$ but... 'cause we're graphing, the input is $\lambda$ the output is the kinetic energy max, so we'd have $K E=1 / \lambda$, well some expression over $\lambda$ because it varies how much of sorta a dip that has. But the basic one would be something over $\lambda$.

The object here is mathematical (a symbolic expression) and both Ben and Amanda's reasoning is primarily 


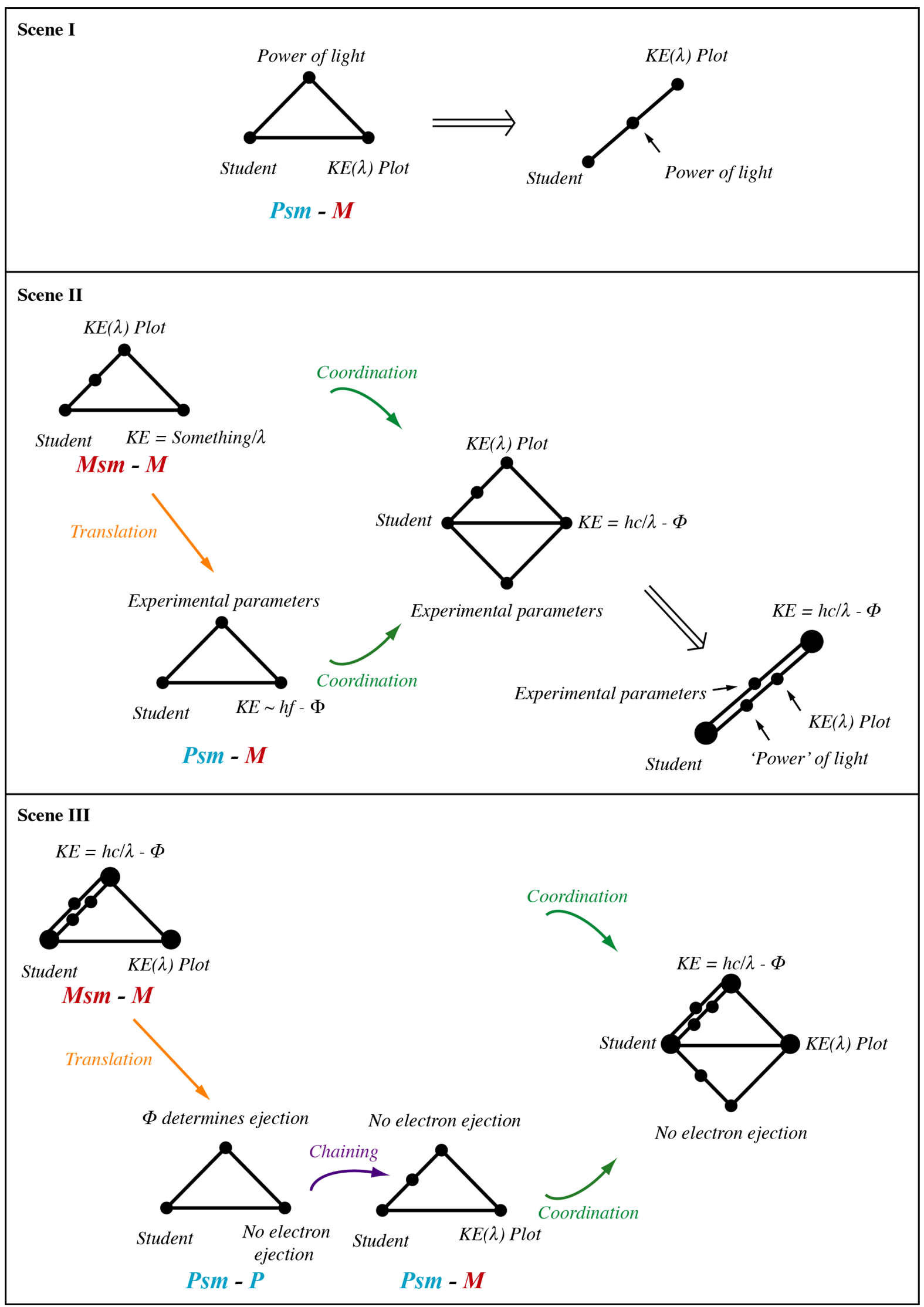

FIG. 9. A sense making diagram for the analysis of the three scenes of case study I. 
mathematical in nature: Ben arguing that it should be a $1 / \lambda$ relationship because of the shape of the graph, and Amanda treating $\mathrm{KE}$ and $\lambda$ not as physically relevant constructs but as mathematical variables (inputs and outputs). In applying mathematical reasoning to a mathematical object, these students are engaged in Msm-M. While it is somewhat straightforward to classify this reasoning as Msm-M when considered in isolation, when the groups' previous work is taken into account it becomes more complicated.

While mathematical in nature, their reasoning about the equation relies on their previously constructed plot of $\mathrm{KE}$ vs $\lambda$. The first notable change from their previous reasoning is a shift in the object of focus. This shift is an example of what Bajracharya et al. [11] would call an interclass representational translation, where students transition from a graphical representation to a symbolic one. We agree with this classification, but argue that this description alone does not fully capture the shift in the students' reasoning. The change captured by this language is a shift in the representational form of the object (noting that both the graphical and symbolic representations are taken to be mathematical in this case), however, there is also a shift in the tool. Previously the students were employing physical reasoning as a tool to generate and understand a graphical object. Now mathematical reasoning regarding this graphical object has become the tool that they employ to understand a new mathematical object, the symbolic equation. As discussed in Sec. II B above, this particular shift in reasoning structure (where the object of focus becomes the tool of future reasoning) is an example of chaining. We do not view chaining as being inconsistent with Bajracharya et al.'s description of representational translations, rather chaining describes a more specific shift in reasoning that simultaneously considers both the tool and object of reasoning. In Fig. 9 chaining is indicated in the first triangle of scene II, where the node on the left leg of the triangle represents the chaining of the prior reasoning mode from scene I.

Following this exchange, the students attempt to determine what the "something" over $\lambda$ should be. They collectively throw out ideas like B: "the work function maybe?" and A: "the voltage would come into play here... it would increase the kinetic energy." At this point the interviewer clarifies that $\mathrm{KE}_{\max }$ refers to the instant the electrons are ejected, which prompts the following exchange:

J: So that'd be hf minus the work function, is the maximum energy it has when it is released from the plate?

B: I guess that would make sense... If you replace this with $h f$, or let me rewrite this [writes $(h f-\Phi) / \lambda$ ]. Then this would follow an inverse relationship and for a larger work function shift it to the left.

J: Yeah, that looks like it works... 'cause the only thing changing is the $\lambda$ value.
The exchange above highlights a translation in the students' reasoning to a Psm-M mode. In contrast to the translation above (chaining) where the previous object becomes the tool, in this translation the object of focus remains the same: the students are still considering a symbolic equation representing the same situation. The primary change here is a shift in the tool. In trying to determine what should be included in the expression they are considering physically relevant constructs (the work function, the voltage, the photon energy). The students do not start with equations that link the relevant variables, rather they are considering what aspects of the physical system could possibly relate to the energy of the electrons. This type of reasoning is notably different from the pattern matching students often use when deciding on an equation to use [41]. This translation is represented by the orange arrow in scene II of Fig. 9. It is worth noting that the students' expression is not correct at this point, as Amanda later notes and corrects:

A: We're like overcomplicating this here.

$J$ : Because $f$ simplifies you mean?

A: Well, we're assuming we were taking this equation $[h f-\Phi]$ and then just putting it on top of this $[\lambda]$ because of the relationship we saw... but because $f$ is defined by $[\lambda]$... if we just rewrite that so, $f=c / \lambda$. Then we have $K E=h c / \lambda-\Phi$.

$J$ : Yeah, and then there's the relationship

A: And then ... $[h c / \lambda]$ would give us the shape of this graph and then that $[-\Phi]$ would give us our shift based on the work function like we were saying before.

Throughout this scene, the group has been engaging in two different modes of reasoning: a chained Msm-M mode and a Psm-M mode. At this point Amanda combines these two modes of reasoning. From their engagement in Msm-M the group agreed that the expression for the kinetic energy must include an inverse dependence on $\lambda$. Additionally, through their engagement in Psm-M, the group has agreed that this kinetic energy should be the difference between the photon energy and the work function $(h f-\Phi)$. As Amanda notes above, they had previously directly combined these two expressions instead of noting the $1 / \lambda$ dependence already present in the frequency. By combining the results of the two modes of reasoning, the students are able to arrive at the correct expression for the kinetic energy, with an understanding both of the functional dependence and of why the expression includes the terms that it does.

The two reasoning structures the students use here (Msm-M and Psm-M) share the same object: a symbolic expression of the kinetic energy. Thus, the combination of these modes is an example of coordination, represented by the green arrows in scene II of Fig. 9. The outcome of this coordination is a reasoning structure represented as a square, which shows the two distinct tools students are using to understand the object. In Fig. 9 the upper triangle represents the chained Msm-M reasoning mode through 
which the students use their plots to determine there should be an inverse dependence on $\lambda$, and the lower triangle represents the Psm-M reasoning mode through which the students determine the dependence of the kinetic energy on physical parameters of the system. In scene III, the students use their equation (the object of scene II) as the tool for further reasoning. For clarity, as in scene I, a collapsed representation of the coordinated reasoning structure showing the students' understanding of the equation is shown. This adds a node in the link between the students and the mathematical equation, showing the chained use of the power of light and the plots in understanding the equation. It also adds a second link between the students and the equation, showing their use of the physical parameters of the experiment in determining what the equation depends on.

In coordinating these modes, the students come to mutually agree on their symbolic equation. Jack notes that it includes "the relationship" that gave them the desired inverse shape and Amanda notes that it includes the "shift based on the work function" that they relied on in comparing the two plates. Mathematically, however, their equation is still inconsistent with their plots, as the expression $h c / \lambda-\Phi$ includes negative values.

\section{Scene III: Synthesis}

In response to Amanda's summarizing statement above, the interviewer then asked the group how $\Phi$ shifts the graph. In answering the interviewer's question, the group encounters a contradiction between their formal mathematical expression and their understanding of the photoelectric effect. To resolve this contradiction the group rapidly shifts between three modes, involving a translation, chaining, and eventually coordination. To highlight these rapid shifts, the coded modes are explicitly noted in the transcript below. The three processes (translation, chaining, and coordination) are shown in scene III of Fig. 9.

Msm-M:

A: Normally in a shift graphically it shifts your asymptotes too... But in our graph we decided that it wasn't going to shift the asymptotes... But then if this [their equation] is right then it would mean that the horizontal asymptote would shift down as well.

\section{Psm-P:}

$\boldsymbol{B}:$ But wait, if the work function is stronger then the electrons wouldn't have any kinetic energy.

A: That's true it would eventually go to zero, it would make the kinetic energy negative but

B: I don't think that's possible yet, gotta wait till tunneling ...

A: Well, eventually, eventually ... no electrons will be coming off the plate once $\lambda$ gets long enough. It won't have enough energy.
Psm-M:

J: Oh yeah, so it'll just drop off... they'll [the graphs] just end.

A: Mhmm. Which would be based on that work function... Once $\lambda$ gets big enough and it has no kinetic energy because nothing is being ejected... And then the larger work function would still be up here [asymptote at infinity for $\lambda=0 \mathrm{~J}$ and it would kinda hit like that [smaller $\lambda$ intercept]... And then this equation could describe it because it would be shifting that graph down but KE always has to be positive so the graph would just end where it intercepts that axis.

B: Yeah, sounds about right. It's more scientifically accurate.

Scene III opens with Amanda employing their equation as a tool to reconsider the plot of KE vs $\lambda$. Taken alone this is an example of Msm-M, where a mathematical equation provides a resource for considering the effect of a given quantity on a plot. In considering the previous work of the group this is not as an isolated instance of Msm-M, but a chaining of an already complex reasoning structure used to reconsider a mathematical object. Using purely mathematical reasoning Amanda discusses how the equation suggests that the asymptotes of their graphs should shift, in contrast to their earlier reasoning about the photoelectric effect. To address this inconsistency Ben shifts the group to a Psm-P mode, using physical reasoning about the experiment to argue about the experimental outcome of a larger work function. Amanda joins in this mode of reasoning, and Ben and Amanda establish that rather than leading to negative kinetic energy a larger work function would lead to no ejection of electrons, and so zero kinetic energy. In comparing the Msm-M and Psm-P modes, both the object and the tool have changed entirely; this shift does not yet build on their previous reasoning, so it is a translation as indicated by the orange arrow in Fig. 9 .

Having used physical reasoning in considering the physical experiment the group has established that the kinetic energy should never be negative. They then enter a Psm-M mode by chaining this reasoning (the purple arrow in Fig. 9) and applying it to their initial plots. This is where Jack notes that the graphs should "just end" and Amanda crosses out their initial plots, replacing them with their final (correct) answer shown in Fig. 8. Building on Amanda's statement that their equation can describe the plots and the physical situation, the interviewer asked if their expression is valid everywhere. This question prompted the following exchange between Amanda and Ben:

A: No. It would only be valid for the interval where the work function is less than. Or the value of $\lambda$ is. Wait... $\boldsymbol{B}$ : When $h c / \lambda$ is greater than or equal to $\Phi$, that's when the expression works. Otherwise you might have a negative kinetic energy at a large enough wavelength. 
This final statement involves the coordination of a repeatedly chained and coordinated Msm-M reasoning structure and a chained Psm-M mode. Ben draws on the mathematical reasoning that the equation does shift the asymptotes, and the physical reasoning that the plots end due to no ejection, to determine the point at which their expression no longer properly represents the physical system. Further evidence for this coordination comes from Ben's statement that their modified plots and equation are more "scientifically accurate," suggesting that there is a strong relationship between the mathematical objects and the physical phenomena they describe. When taken alone, this reasoning might be considered an example of Psm-M, where physical criteria are used to understand the bounds of a mathematical expression. However, a deeper analysis shows this reasoning can be viewed as a complex molecule of sense making that relies on repeated chaining and coordination of multiple individual reasoning modes.

\section{CASE STUDY II: PENNY, MORGAN, CAM, AND SARAH}

This second case study, as discussed in the methods section above, draws on student data from a separate course, in a different semester, with students considering a question with different content. Below we briefly discuss the prompt but remind the reader that the authors played no role in instruction for this course. As such, we cannot comment on the students' prior exposure to this physical system, other than to note that finite wells and tunneling were included on the course syllabus. As with the previous case study, the analysis of the reasoning episode is broken into three scenes, but here we also include a "prologue" and an "epilogue."

\section{A. Context and prompt}

In the portion of the focus group presented here, the students are considering a question adapted from Griffiths

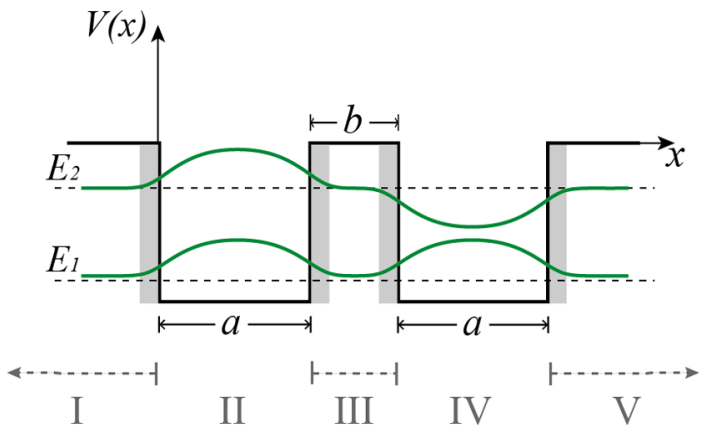

FIG. 10. A plot of the double finite square well potential. The regions were indicated by $\mathrm{Cam}$, and were not present in the initial diagram. Sketches of the ground state and first excited state wave functions (the answer to the protocol question) are shown on top of the potential.
[42], regarding a symmetric, double, finite square well. The two wells are each a width $a$, have a depth $V_{0}$, and a variable well separation $b$. A diagram of this potential is shown in Fig. 10, though the five different regions were indicated and labeled by Cam and were not present in the figure given to the students. The question asked for a sketch of the wave functions for the ground state and the first excited state of the well, for three values of the well separation: $b=0, b \approx a$, and $b \gg a$. Sketches of these wave functions for $b \approx a$ are shown on top of the potential in Fig. 10, and were also not present in the figure provided to students.

\section{B. Prologue: Recall}

In beginning to consider the problem, Cam and Morgan immediately note that because the wells are finite there is the possibility of tunneling. As they continue on, Cam indicates and labels the five regions of the potential (as shown in Fig. 10) and suggests that the wave function can be broken up into separate pieces that are smooth and continuous at the boundaries. He states that in each of these regions the wave function should be either a sine or a [real] exponential, though he gives no justification for this assertion. Morgan brings up the idea of normalization, saying that the wave function must "integrate from negative infinity to infinity of one." Morgan uses normalization as justification for why the wave function in regions I and $\mathrm{V}$ should be real exponentials, but is less certain of the behavior in region III:

M: The ones at the ends [RI \& RV] have to be exponentially decreasing, that's always going to be true. The real question is what happens with the one in the center [RIII]? Because we could model that as exponential decrease from one direction to the other and then have there be a sine wave in here [RII \& RIV] ... But, I guess, do we just arbitrarily pick a direction for it to exponentially fall off? ... I dunno if we can do this but nothing about the regularity [boundary (?)] conditions seem to indicate that we can't have this be a sine wave in here [RIII] ... I understand that seems wrong but like, intuitively that seems wrong I just have no mathematical justification for why it's wrong.

$P$ : Could they be, are you saying that there would be, it would be the same sine function or that it would be a sine function that's different from the ones in here [RII \& RIV]? ... It seems like it should be different.

M: It does seem like it should be different.

This exchange sets up the remainder of the focus group discussion. Much of the reasoning presented so far, while necessary for the work that follows, would likely not be considered sense making on its own. Save for Morgan's reference to the "regularity conditions"- a term we have not heard before, but believe refers to the boundary conditions on the wave function: e.g., continuity, and 
normalizability - Cam and Morgan have offered no reasoning for the many assertions they have made. While these statements (the wave function should be smooth and continuous, tunneling is possible, the wave function must integrate to one, the solutions are sines and exponentials) are largely correct, they are disconnected, unsupported facts that we argue are recalled rather than understood by the students. That said, Morgan has begun to define what it is the group should figure out (i.e., an object of sense making), specifically the form of the wave function in region III. The transcript above suggests that the students have an intuitive sense that the region between the wells should exhibit different behavior than the regions inside the wells, and to address this intuition they spend the remainder of the reasoning episode developing a "mathematical justification" for the form of the wave function in region III.

\section{Scene I: From $\psi$ to Schrödinger}

Having settled on a goal-a mathematical explanation for the wave function in Region III-the interviewer asks the students to talk about where the two types of solutions they have been discussing come from, and why they might be valid in one region and not another. Cam and Morgan reiterate that outside the well they have to go to zero so that the wave function is normalizable, and then Morgan and Penny continue on to recall a simplified Schrödinger equation:

M: I mean, where the sine and the cosine come from, I'm not sure if that's what you were asking, but they're solutions to a differential equation... Is it the Schrödinger equation that they're solutions to? I'm not sure I completely remember which one... No! They're solutions to the wave equation?

$\boldsymbol{P}$ : There's like a double differential equation [writes $\left.\frac{\hbar}{M} \frac{\partial^{2} \psi}{\partial x^{2}}=-\square \psi\right]$

$S:$ Yeah, I think that's Schrödinger.

M: ... And that's the differential equation, its solutions include $e^{k x}$ and $\sin (k x)$ but uhm it might have more solutions that we don't know about...

P: Because, the only way we were given, like they, in the book they like develop this equation... And then it was like [waves hands mockingly] this is a solution.

M: We were just given "here are two possible solutions, in this class we're always gonna have one of these be the solution" but there might be others. We haven't talked about that.

Above we claimed that much of the students' reasoning had been largely recall, and this excerpt provides clear evidence of this. Morgan states that they do not remember which differential equation the wave function solves, and while Penny recreates a rough template of the Schrödinger equation (line A in Fig. 11) the group offers nothing to

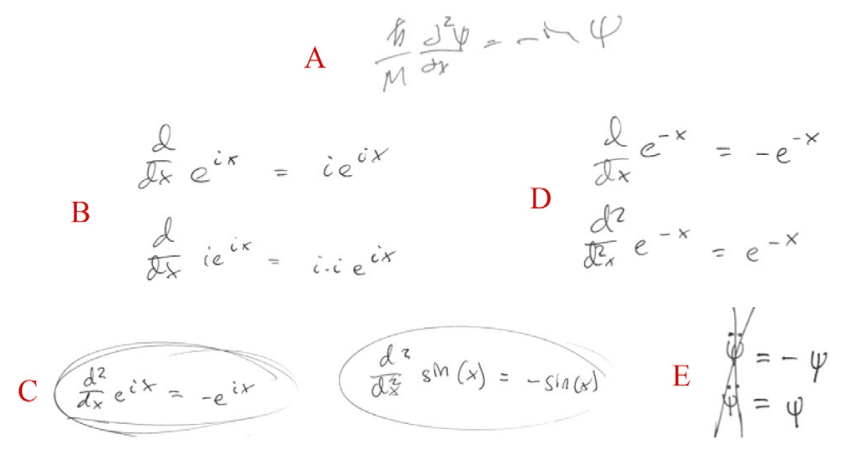

FIG. 11. Student work by Penny and Morgan. The letters show the order in which this work was written and are used for reference in the text.

suggest an understanding of the meaning behind it. In fact, the portion of the Schrödinger equation that determines which of the two solutions (real or complex exponentials) is valid Penny has left as a blank. Penny and Morgan's final exchange solidifies that the students are using memorized solutions, but do not know when or why these solutions are valid.

Immediately following this, Penny brings up Euler's formula, asking "where does like, the imaginary thing come from? How, ya know like, sine cosine with something $i$ equals $e^{i}$." After writing down the correct form of Euler's formula $\left[e^{i \theta}=\cos (\theta)+i \sin (\theta)\right]$, the group plugs the complex exponential into their template Schrödinger equation (meaning they take two derivatives). This portion of the student work, written by Morgan, is shown in line B of Fig. 11. After taking these derivatives Morgan writes line $\mathrm{C}$ of Fig. 11 while saying that "the second derivative with respect to $x$ of $e^{i x}$ is equal to negative $e^{i x} \ldots$ It's also true of sine, right? ... The second derivative of $\sin (x)$ with respect to $x$ is negative $\sin (x) \ldots$ Sooo, that's why those two $\left[e^{i x}\right.$ and $\sin (x)]$ are the solutions to that differential equation [Penny's template Schrödinger equation]." The interviewer asks if this is also true of real exponentials, which leads Morgan to take more derivatives (line D of Fig. 11), at which point Penny exclaims "so like the second derivative equals. Ooooh, it doesn't equal negative $\psi$." To summarize their work, Morgan writes the two simplified differential equations shown in line E of Fig. 11. Morgan crosses out the LHS after a brief discussion that dots generally indicate derivatives with respect to time. Morgan later expands on this work, as shown in Fig. 13, using primes rather than dots to indicate derivatives with respect to space.

In this scene the students begin to engage in sense making rather than recall. The students know that there are two types of solutions and have admitted that they have memorized these. As Penny and Morgan noted, they are not sure if these are the only solutions to the Schrödinger equation, or when they are valid. However, they use these memorized functional solutions as a tool to address exactly these questions. By taking derivatives the students begin 
to understand, and recreate, the Schrödinger equation. This purely mathematical process involving symbolic manipulations used to understand a symbolic mathematical object (the Schrödinger equation) is Msm-M, as indicated in scene I of Fig. 12. Again, Fig. 12 shows the complete sense making diagram for the entire reasoning episode, including all three scenes and the epilogue.

The structure of Msm-M reasoning here is different than the Msm-M mode discussed in the previous case study, where Amanda used a symbolic equation as a tool to understand a graphical object. Though both the tool and object of sense making are categorized as mathematical in both cases, Amanda's reasoning involved two separate representational forms (interclass) while here the students employ a single representational form (intraclass) to do "just math." While the distinction was not necessary before, in the remainder of this analysis it will be critically important to distinguish between different Msm-M structures. To do so, we suggest that each reasoning mode has multiple flavors that depend on the specific entities used as the tool and object, as well as their representational form. Due to the vast number of combinations this creates we do not label each of these flavors. However, we do extend the process of translation to include any change in the tool or object. Thus, translation can occur between different modes (e.g., Msm-M to Msm-P) or between different flavors of the same mode.

The particular flavor of Msm-M discussed here involves only symbolic representations of mathematical entities, and other frameworks might label this type of work as process [4] or execution [5] where students engage in formal mathematical manipulations with no direct connection to any physical system. This turn-the-crank or plug-and-chug style of mathematical reasoning has often been singled out as explicitly not sense making [18,19,21]. However, we argue that these students are engaged in MSM in a way that is both rich and productive in understanding the physical system of the double square well.

An argument might be made that the prologue should be a scene like the others. However, we see scene I as a turning point where the students begin to engage in sense making, building further understanding from their previously memorized knowledge. The recall in the prologue was a necessary step for this sense making, as it generated resources for these students to begin to fit together, but it was not until scene I that they truly began to "figure something out." Throughout this phase of their work, the students have abandoned any direct (explicit) connection to physical quantities. The Schrödinger equation has been simplified to a math problem, with several physically relevant constants jumbled together or dropped entirely. As indicated by their work in Fig. 11, the group has come to see that the two types of wave functions are the solutions to two different differential equations (differing by a minus sign). They have not yet indicated where this minus sign comes from, and so have not discussed when the two types of solutions are valid.

\section{Scene II: From Schrödinger to $\psi$}

The group has now agreed that both types of solutions are valid in general but have not discussed when each of the solutions should be used. In an attempt to encourage this discussion, the interviewer asked the group "what has to be different in the Schrödinger equation to use exponentials versus sines and cosines?" which led to the following:

M: ... It has to do with energy somehow. Like that's why, right? If it's going to decay out here [RI \& RV] it's decaying because the energy ... The Schrodinger equation has like a ... some constant, I don't care what it is ... the second derivative of $\psi$ equals like $U(x)-E$ or something like that? Maybe I have that swapped around?

C: Yeah. I think it's $E-U(x)$

M: ... So basically, it's some constant here, some value here, it could be a constant ... So it's a combination of these two differential equations [line E in Fig. 11]. I think. So that's why there's both solutions...

JG: What was your condition for which solution you pick out of the Schrödinger equation?

$M:$...When the energy. Oh. Oooh, when it's this thing. When this $[E-U(x)]$ is positive.

Though Morgan begins their response in what seems to be a mode of physical reasoning, discussing "the energy," they do not actually engage in physical reasoning regarding the energy. Rather, they return to the Schrödinger equation, discussing "some constant" and "the second derivative of $\psi$ " which equals " $U(x)-E$ or something." Despite the physical relevance that may be present for researchers and experts when using constructs like energy, the argument made by Morgan involves primarily mathematical reasoning. Morgan continues on and, with a brief prompt, is able to connect the differing sign in their two equations (line $\mathrm{E}$ in Fig. 11) to the sign of $E-U(x)$. Scene II of Fig. 12 represents this reasoning, indicating how Morgan is using their two simplified Schrödinger equations as a tool to generate and understand the complete Schrödinger equation. Morgan is still engaged in Msm-M, using a symbolic mathematical tool to understand a symbolic mathematical object, but as the object and tool have changed from before this constitutes yet another flavor of Msm-M. In particular, Morgan's current reasoning can be understood as a chaining of their previous reasoning, indicating that the process of chaining can occur both between different modes and different flavors of the same mode. Morgan has now indicated the relevant piece of the Schrödinger equation ["the $E-U(x)$ " which they treat as a mathematical entity]; in the following excerpt Penny attempts to apply physical meaning to this mathematical term. 


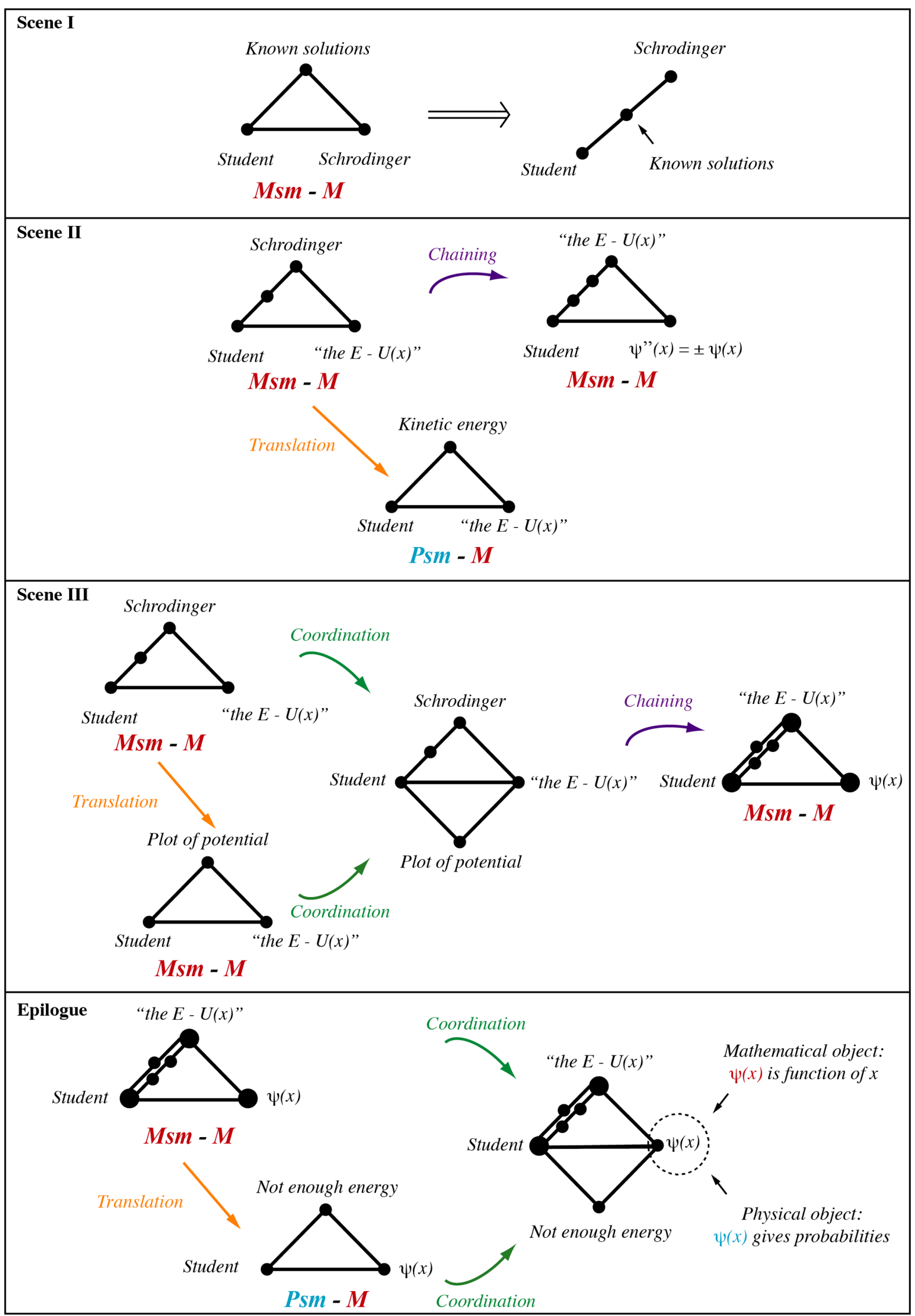

FIG. 12. A sense making diagram for the analysis of the three scenes, prologue, and epilogue of case study II. 
P: What is $E-U(x)$ ?

M: ... So basically, $E$ is the energy of the particle, I think $\ldots$ and then $U(x)$ is the potential as a function of $x$.

P: So $E-U(x)$ is like the kinetic right?

$S$ : Yeah, should leave kinetic.

M: Right. But. So, if $U(x)$ is bigger than $E$, then the kinetic energy is weirdly negative, uhmm and I think. So, if we have. If it is negative then, constants aside, we have that equation $\left[\psi^{\prime \prime}(x)=\psi(x)\right]$. If it's positive we have, constants aside, that equation $\left[\psi^{\prime \prime}(x)=-\psi(x)\right]$.

Previously the group has repeatedly chained several flavors of Msm-M, using the mathematical solutions to determine a difference in overall sign in the Schrödinger equation and then determining that this sign comes from a particular term in the Schrödinger equation. Now, in an attempt to combine this mathematical reasoning with physical reasoning Penny asks a question that translates the group (briefly) to a Psm-M mode. Rather than focusing only on whether a mathematical entity [the $E-U(x)$ ] is positive or negative, Penny also wants to know what this constant means in the context of physics. By engaging in physical reasoning, she attempts to interpret this mathematical object as a physically relevant quantity: the kinetic energy.

Though Morgan agrees with Penny that $E-U(x)$ should be the kinetic energy, they do not attempt to further integrate this idea into their discussion. Instead, after stating that the kinetic energy would be "weirdly negative" Morgan chooses to abandon this physical line of reasoning and instead returns to an Msm-M mode where there is no issue with this mathematical constant being negative. In this Msm-M mode, Morgan engages in another step of chaining to use the $E-U(x)$ as a mathematical tool to determine the form of the Schrödinger equation, and so the functional form of the wave function. While Penny has made a bid for coordination between a physical (Psm-M) and mathematical (Msm-M) understanding of the $E-U(x)$, this bid has been abandoned by the group and the $E-U(x)$ remains a mathematical entity whose physical significance is overlooked. As further evidence that coordination of these modes does not occur, throughout the remainder of their discussion the group continues to say the $E-U(x)$ rather than "the kinetic energy."

It is relevant to note that it is not simply a linguistic difference in the label kinetic energy versus the $E-U(x)$ that suggests Penny's attempt at physical reasoning, but also the way that these students are treating this quantity. In calling it kinetic energy the group interprets it as a physical quantity for which a negative value would be "weird"meaning inconsistent with their experiences with models of the physical world. By returning to a label of the $E-U(x)$ the group discards this conflict and treats it as an abstract mathematical entity that has no physical restrictions on its sign. While we acknowledge the messiness in coding this entity, we reiterate that our codes are centered on how the entity is being treated by the learners rather than solely on an expert interpretation.

In scene II the dominant reasoning structure is the repeated chaining of Msm-M modes. As shown in Fig. 12, this scene ends with a reasoning structure that uses the $E-U(x)$ as a mathematical tool to determine which version of their Schrödinger Equation is relevant (and so what the wave function should be). Getting to this point does not require Penny's bid to engage in Psm-M and, since this mode is not coordinated with the rest of their reasoning, this brief shift is a translation. Though the group does not take up Penny's bid for coordination (and arguably Penny also abandons this line of reasoning as well), we are not suggesting that this attempted coordination is irrelevant. On the contrary, as discussed in another paper [39], Penny's repeated bids (taken up or not) are often the driving factor in the group's sense making. We view it as a benefit of the framework that it highlights the processes by which reasoning structures are combined and coordinated and also indicates instances when shifts in reasoning do not lead to coordination with other reasoning modes.

\section{E. Scene III: the $E-U(x)$}

With her previous bid for coordination abandoned, Penny joins Morgan in a Msm-M mode, asking for clarification about how to use the $E-U(x)$ as a mathematical tool to determine the wave function. During Morgan's monologue below they are writing the first three lines shown in Fig. 13. Though the transcript is not included here, as shown in Fig. 13 Morgan continues beyond the quoted text to indicate the conditions for both types of solutions.

P: So what does the $E-U(x)$ have to do with the, what $\psi$ can be?

M: So if we have a Schrödinger equation which looks essentially like negative a positive constant times the second derivative of this $[\psi(x)]$ uhh, equals $E-U(x)$ times that $[\psi(x)]$. So if $E-U(x)$ is negative, is less than zero, right this $[E-U(x)]$ is negative this $[-k]$ is negative, so we get essentially the solutions to that differential equation $\left[\psi^{\prime \prime}(x)=\psi(x)\right]$.

$$
\begin{aligned}
& -k \psi^{\prime \prime}(x)=(E-U(x)) \psi(x) \\
& \text { if } \begin{aligned}
E-U(x) & <0 \\
\psi^{\prime \prime}(x) & =\psi(x) \rightarrow e^{-x}
\end{aligned} \\
& \text { if } e-U(x)>0 \quad \ldots . . . . \\
& \psi^{\prime \prime}(x)=-\psi(x) \rightarrow e^{-i x}=A \cos (x) f \sin \left(x^{\prime}\right) \\
& \psi^{\prime \prime}(x)=-\psi(x)
\end{aligned}
$$

FIG. 13. Student work (written by Morgan) summarizing the possible solutions to the Schrödinger equation for constant potentials. 
In the above Morgan links the sign of the $E-U(x)$ to the known solutions of the Schrödinger equation. Again, despite the previous relation to the kinetic energy, in this moment there is no evidence that either the $E-U(x)$ or the wave function itself has physical significance to Morgan. However, this lack of explicit physical interpretation does not automatically preclude the group from engaging in MSM as other definitions that suggest a constant blending of physical reasoning and mathematical formalisms might suggest. On the contrary, we argue that this reasoning episode exemplifies rich MSM in which the students are engaged in the construction of an explanation that helps them understand the correct functional form of the wave function. This reasoning involved developing an understanding of the mathematical structure of the Schrödinger equation, and can be described as a repeated chaining of Msm-M modes. While this math-only reasoning structure might not be the preferred end goal of student understanding, i.e., we hope our students will eventually coordinate this with physical reasoning, we argue that this extended math-only reasoning episode was an important, fruitful step towards understanding the double well system. We see the ability to describe "pure" MSM and its possible connections to physical reasoning to be a defining characteristic of the framework.

In another bid to understand where the sign of the $E-U(x)$ comes from, Penny asks

\section{P: How does, uhm, the I guess like location along $x$ determine whether $E$ is like $E-U$ is less than zero or greater than zero? \\ M: $E$ is constant, but $U(x)$ is a function of $x$. \\ $C$ : Yes. And so yeah, you could look at the potential [indicates graph]. \\ M: So as $x$ changes this [the potential] is gonna change \\ $P$ : Is gonna change from being zero to being \\ $P \& C: V_{0}$}

Previously Morgan has related the form of the wave function to the sign of $E-U(x)$. Here, due to Penny's query, the students begin to address where the sign of $E-U(x)$ comes from. Penny's language ("location along $x$ " rather than "position" or "where we are in space") though closer to considering the physical context is still mathematical in nature, as is Morgan's statement that " $U(x)$ is a function of $x$." Given this, the students are still engaged in an Msm-M mode, however Cam's statement suggests that it is a different flavor of Msm-M than before. By saying that they could "look at the potential" and explicitly indicating the graph, Cam shifts the group into using the plot of the potential as a tool to understand how the sign of the $E-U(x)$ is determined. Since the mode does not change in this translation, as indicated in scene III of Fig. 12, this is a translation between flavors rather than between modes.

In what follows, Morgan combines these two flavors of Msm-M, leading to a coordinated understanding of the
$E-U(x)$ that leverages both the plot of the potential and the Schrödinger equation as tools. They then use the $E-U(x)$ as a tool (another step of chaining) to determine the wave function in region III.

M: So it does have to be the decaying one in the middle. It can't be a sine wave. Well that's nice.

$\boldsymbol{P}$ : Wait, why?

M: Because here we have the, we're in this $\left[\psi^{\prime \prime}(x)=\psi(x)\right]$ condition, because E minus the potential, the potential is high up here [indicates value at top of wells] and we presumably don't have the energy.

P: Yeahhh. I mean. I dunno what E is. What determines, so I guess the thing that determines $E$ is $n \ldots$

M: Right. But like, that, that. This observation. So like when $U(x)$ is high, above what we think the energy of our electron is then we know that uhh $\psi(x)$ has to take on a form that's a negative exponential. Or a positive exponential, but the positive exponential can't happen because of regularity conditions.

The group admits that they don't know what the energy of the particle is, relating it to the quantum number $n$ and the formula for the energies of the infinite square well that they do not remember. However, throughout the focus group they have been treating these wave functions as bound states-requiring that the wave function go to zero at $\pm \infty$. This is in line with the protocol prompt, which asks for the wave functions for the first two energy states; language that implicitly assumes bound rather than scattering states. Penny's concern about the value of energy is only tangentially addressed, as Morgan hedges saying that the wave function should be an exponential when the potential is above what they think the energy of the particle is.

This reasoning episode began when Morgan set out to find a "mathematical justification" for their intuition that the wave function should be different in Region III than in II or IV. Having found this justification, they seem to be satisfied. We note that this is not actually a complete answer to the interview question, which asks for sketches of the ground state and first excited state wave functions, not the general functional form in each region. In the remainder of the focus group, the students make progress towards the correct answer, posing and attempting to answer questions including "which well does it start in?" and "should it be equally in both?" However, we end our analysis here to focus on the students' dominant engagement in differing flavors of Msm-M. In the three scenes of this reasoning episode the students have generated a complex molecule of reasoning, as shown in Fig. 12, in which the only bid for explicit physical reasoning was abandoned. Despite the group's almost exclusive use of mathematical reasoning, this reasoning episode shows important and productive MSM in the context of physics. This extended period of "math-only" reasoning was a useful step for these students 
that allows them to continue on to address the more physical aspects of the question. This reasoning episode highlights the frameworks' ability to categorize and distinguish between the ways students can use math when reasoning about physical systems. It also suggests that there are instances where pure mathematical reasoning can be a productive step in physical sense making. Perhaps this is an obvious claim, however it sits in contrast to some definitions of mathematical sense making that view MSM in physics as the constant blending of conceptual physics and formal mathematics $[18,20]$.

\section{F. Epilogue: Intuition}

Though Morgan seems satisfied with their work, again Penny goes beyond the shared group reasoning in an attempt to coordinate the group's purely mathematical reasoning with her physical intuition. The two lines below come immediately after Morgan's final statement above, where they argue that the wave function should be a decaying exponential when the potential is greater than the energy.

$\boldsymbol{P}$ : And then that's, that is intuitively right. That if the energy is, if the potential energy is higher than the energy of the thing then the thing is not like as likely to be there.

M: Right, well, yeah it drops off exponentially as you get further away from the uh low regions.

Though this exchange is brief, we argue that it shows Penny coordinating the group's mathematical result (that the wave function is a decaying exponential when $V>E$ ) with a physical interpretation of the wave function (that the amplitude tells us where the electron is likely to be found, and that this should be lower when "it doesn't have the energy to be there"). This involves a brief translation from an Msm-M mode to a Psm-M mode, and then a coordination of the two-as shown in Fig. 12. Penny made a similar bid for coordination in Scene II when she attempts to interpret the $E-U(x)$ as the kinetic energy, a bid that was ultimately abandoned by both Penny and the rest of the group. In contrast, here Penny effectively coordinates these two reasoning modes. We argue, however, that while Penny coordinates these modes the group as a whole does not fully take up her bid. The language of Morgan's response, that "it" drops off exponentially, suggests that they have remained in an Msm-M mode-simply stating the shape of the function. This response does not address the deeper meaning of Penny's statement, which involves a conceptual argument about the physical behavior of an electron.

As this exchange is the closest to physical reasoning that the group comes during this reasoning episode, one could argue that it fits naturally as the end of scene III. However, while the Msm-M structure developed in the three scenes of the reasoning episode is largely shared by the group as a whole, this coordinated structure developed by Penny is not shared by the rest of the group. As is discussed elsewhere [39], the interplay between cognitive reasoning and social dynamics in this group are complex. Penny is often the group member that drives the sense making, asking questions and providing bids for coordination and connection between mathematical and physical reasoning. These bids are often not taken up by the rest of the group. This epilogue highlights this disconnect, suggesting the continued sense making Penny engages in even when the group does not take up her bids.

This coordination of physical and mathematical reasoning regarding the same object is suggestive of the dual nature many entities have for expert physicists. It is natural here to categorize the wave function as a mathematical object, especially since the group treats it largely as a symbolic solution to the Schrödinger equation. Yet in this epilogue, Penny interprets the physical meaning of the wave function. We have classified this as physical reasoning about a mathematical object (Psm-M); however, Penny's language focuses on the behavior of an electron, which is suggestive of a physical object. As Penny's utterance is so short, there is not sufficient evidence to clearly categorize $\psi(x)$ as either a mathematical object or a physical one. Ultimately, for the experienced physicist, the wave function is likely both a mathematical and a physical entity. This dual view of an entity is an example of a dynamic ontology [43] which is built up over time, perhaps through engagement with coordinated reasoning structures like the one developed in this epilogue.

\section{DISCUSSION AND CURRICULAR IMPLICATIONS}

In both of the case studies presented above, we have argued that a complex, extended activity of mathematical sense making can be viewed as the repeated engagement in and combination of four basic sense making modes. As instructors, it may be sufficient to recognize sense making in our students as an intuitive know-it-when-you-see-it phenomenon. However, if sense making is included as a specific learning goal, greater specificity can be helpful. To provide this specificity, we have attempted to operationalize some of the cognitive processes of MSM by classifying four general sense making modes that describe how mathematics may be used in the context of physics. These modes distinguish between the object of sense making (what is being figured out) and the tool (how it is being figured out).

The four modes are not exhaustive, nor are they "fundamental" in the sense of being the smallest possible grain size. In the analyses above there were many instances where the four modes were sufficient to describe learner reasoning; however, in the second case study, there were many instances where it was critically important to identify different flavors of each mode that differ in the particular 
representation (symbolic, graphical, etc.) of a tool or object. Furthermore, on an even smaller grain-size there may be phenomenological primitives [36] or resources [35] upon which each mode is built. We view it as an important outcome of this current work that the framework can often be productively applied at this middling grain size but is also capable of describing reasoning in finer detail when necessary. To continue with the particle analogy, the four modes are like atoms of reasoning, as opposed to quarks (specific flavors of each mode) or molecules (combinations of the modes). We have opted to take this middling grain size as the basic building blocks in an attempt to study mathematical sense making specifically. In doing so we are not arguing that the finer grain size is unnecessary, as demonstrated in case study II sometimes this finer grain size is critical. However, we do argue that this middling grain size is often sufficient. As with any model, we seek to strike a balance between sufficient accuracy and ease of application.

Though the modes of the framework rely fundamentally on specifying whether an object or tool is mathematical or physical, it is not always straightforward to categorize an entity this way. While we have provided a heuristic for this distinction-mathematics referring to the moves, protocols, and relations between abstract entities and physics being anchored in the phenomenon of the real world-others may disagree with this heuristic. Under a different definition of math and physics the individual codes applied to student reasoning may differ; however, we do not find this difference particularly problematic as the framework still offers a useful tool for considering the reasoning students engage in (or that we want students to engage in)-regardless of the label we assign it. We reiterate however, that we follow Dewey in centering the experience of the learner, through our own lens as content experts.

In this vein, we address a specific issue in classifying entities: to an expert, entities-or specific representations of these entities - often have both physical and mathematical meaning. One could even argue that the ideal goal for students is to see mathematical representations as harmonious with, rather than separate from, physical models. We agree with this perspective. However, we argue that this dual-nature perspective comes from the compilation of complex reasoning structures and must be built up as learners are exposed to novel content. This is in line with arguments by Podolefsky that once ideas have been compiled it takes conscious effort to break them apart [37]. He posits that expert reasoning can be described in terms of compiled structures, and that experts are likely not always conscious of the individual ideas that have been compiled. Thus, while ideal or expert reasoning may involve instances where it is neither possible nor desirable to classify something as purely physical or mathematical, we believe that the framework can still be used as a scaffold to understand student reasoning. In addition, the framework captures instances where students are treating an entity as purely mathematical or physical as well as instances where they are attempting to coordinate these views, e.g., treating the $E-U(x)$ as a mathematical object and choosing not to interpret it as the kinetic energy.

In the analyses above we identified three particularly important processes by which these modes are linked: translation - any instance where the tool or object of sense making changes, chaining - a specific translation where the object of sense making becomes the tool for further sense making - and coordination - where two separate modes that consider the same object using different tools are combined. We do not argue that these are the only processes that can build up a molecule of sense making, only that they are prevalent in the data we have presented and productive in describing student reasoning.

While we argue that student engagement in these modes is not based solely on the specific instructor, content, or type of instruction, we also argue that this analysis suggests a strong role for instruction. In both case studies students engage in translation, chaining, and coordination organically, e.g., when Penny asks what the $E-U(x)$ means or how they can figure out whether it is positive or negative. However, there are also several instances where these shifts in reasoning structure are prompted by the interviewer, e.g., when the interviewer asks where the two known wave functions come from, or if the expression $K E_{\max }=$ $h c / \lambda-\Phi$ is always valid. Whether student driven or instructor driven, these queries prompt the students to engage in different forms of reasoning by shifting the object, tool, or structure of the reasoning mode.

Though these were not framed as teaching interviews, these interviewer interventions can still be viewed as a form of instruction. Thus, these case studies suggest that instructional interventions can successfully lead to sense making by encouraging students to shift between, chain, and coordinate reasoning modes. These processes lead to complex molecules of reasoning at a grain size that intuitively feels like sense making in a know-it-whenyou-see-it fashion. Thus, this categorical framework offers a language for researchers and instructors to consider both student reasoning and instructional interventions-be that in the form of curricular materials or in-the-moment interactions. Though we are not suggesting that the language of this framework be explained to students as a standard problem-solving strategy might be $[1,2]$ we do suggest that instructors might leverage this framework as a foundation for designing curricular activities, lectures, or homework problems. By considering not only the content that we wish students to learn, but also the multiple tools we expect students to use and combine in understanding this content, we argue that sense making can be an attainable, measurable learning outcome.

Attending to shifts in reasoning is not a novel curricular implication in PER. Similar shifts in epistemic games or 
framing have been discussed before [7-9], and more recently the language of inter- and intrarepresentational transformations has been added to the collective discourse [11]. However, our framework exists on a different grain size of reasoning structure than many of these other descriptions of student reasoning. A call to attend to translations implies specific consideration of both the object and tool of reasoning, while attention to chaining and coordination imply consideration of the construction and application of a larger "molecule" of sense making. These molecules of sense making can be consistent with the grain size of epistemic games and frames; so in addition to describing mathematical sense making more broadly, the framework also offers greater specificity to the individual cognitive moves involved in these activities.

\section{CONCLUSIONS AND FUTURE WORK}

Starting from a broad discussion of scientific sense making and the use of mathematics in physics, we have presented a categorical framework for considering mathematical sense making in the context of physics. This framework operationalizes some of the cognitive processes involved in scientific sense making, positing four modes of sense making that differ in the object and tool of the sense making: Use of a mathematical tool to understand a mathematical object, use of a mathematical tool to understand a physical object, use of a physical tool to understand a mathematical object, and use of a physical tool to understand a physical object. Though it is not always possible, or desirable, to clearly define a particular entity as mathematical or physical, it can often be useful to do so. This categorization is not based on an entity being fundamentally mathematical or physical, but rather on how it is locally discussed or applied by the learner.

Each of these four sense making modes have multiple flavors that differ in the specific entity, or representation of that entity, used as a tool or object; for example, while a graph and a symbolic equation may both be treated as mathematical entities, using the equation to understand a physical phenomenon is a different flavor of Msm-P than using the graph to understand the same phenomenon. While engagement in any one of these modes constitutes a cognitive process that could be considered sense making, simply using a particular tool does not necessarily meet the larger definition of building an explanation to figure something out. Often, it is not the individual use of one of these modes that counts as sense making, but the ways students shift between and link these modes. We identify three processes by which the modes are linked: translation (any time the object or tool or sense making changeseither a change between one of the four modes or a change in the flavor of one mode), chaining (a specific translation where prior reasoning units build on each other), and coordination (a blending of reasoning units).
In the two case studies, we demonstrated how the framework can be used to describe and understand extended episodes of student reasoning. These two specific episodes were chosen to highlight the general applicability of the framework - that engagement in these modes is not explicitly tied to a particular course, content domain, or instructional type - and also because they show reasoning that is recognizable, intuitively, as sense making. In our analysis we showed that this know-it-when-you-see-it sense making can be described in terms of the creation of a molecule of reasoning through translation, chaining, and coordination of the four modes. These processes were represented using sense making diagrams that indicate the building of complex reasoning structures through translation, chaining, and coordination. These representations can give a rough sense of how complicated a particular activity may be and could be suggestive of the scaffolding necessary to help learners engage in particular forms of reasoning.

These extended audio and video recorded reasoning episodes provide rich data to analyze with the framework. One benefit of this rich dataset is that it is easier to unpack student reasoning and get an intuitive sense of whether their work counts as sense making. Less rich datasets, for example, written responses or answers to multiple choice questions, do not always allow for the same intuition regarding sense making. In future work we will begin to investigate how, if at all, the framework must be modified and what insights it can provide into student reasoning when it is applied to these coarser grained data streams.

Another application of this framework is the ability to track the frequency of (and opportunities for) use of the various modes of mathematical sense making. If such an analysis had been conducted here, it would have been seen that no instances of Msm-P were observed in the two case studies. We do not take that as an indictment of these students or of the curricula, rather we see this as a natural response to the specific framing of the tasks presented here: in both case studies the object of focus is mathematical (plots of KE vs $\lambda$ or the wave function), and predictions of physical phenomenon were not specifically requested. A forthcoming paper will explore the frequency of modes in greater depth, and the effect of specific cueing on these frequencies.

The analysis here is also suggestive of an approach to address specific goals of instructional interventions. We have shown that particular interviewer interjections can lead students to alter their reasoning structure, e.g., translating students into a different mode or encouraging them to chain or coordinate their prior reasoning. Experienced teachers often have an intuition for the question or instructional intervention that may help students, though (as with recognizing sense making) perhaps not always a specific explanation for why the particular intervention will help. We believe that this framework can provide specificity in describing the goals and effects of certain instructional 
strategies while still valuing the intuition of experienced teachers. In addition to describing instructional techniques, we also feel that this framework can be used as a guide for the design of curricula. The application of this framework to curricular design will also be the subject of future work.

\section{ACKNOWLEDGMENTS}

The authors would like to thank the students that participated in these focus groups. We are grateful for discussions with and the prior work of Joe Redish, feedback from our collaborators in the PER groups at the University of Colorado Boulder and the University of Maryland (in particular Jessica R. Hoehn and Steven J. Pollock), and the detailed and constructive feedback of anonymous reviewers. This material is based upon work supported by the National Science Foundation Grants No. DUE 1625824 and No. DMR 1548924. Any opinions, findings, and conclusions or recommendations expressed in this material are those of the authors and do not necessarily reflect the views of the National Science Foundation.
[1] J. I. Heller and F. Reif, Prescribing effective human problem-solving processes: Problem description in physics, Cognit. Instr. 1, 177 (1984).

[2] A. Van Heuvelen, Learning to think like a physicist: A review of research-based instructional strategies, Am. J. Phys. 59, 891 (1991).

[3] L. Hsu, E. Brewe, T. M. Foster, and K. A. Harper, Resource letter rps-1: Research in problem solving, Am. J. Phys. 72, 1147 (2004).

[4] T. J. Bing and E. F. Redish, Analyzing problem solving using math in physics: Epistemological framing via warrants, Phys. Rev. ST Phys. Educ. Res. 5, 020108 (2009).

[5] B. R. Wilcox, M. D. Caballero, D. A. Rehn, and S. J. Pollock, Analytic framework for students' use of mathematics in upper-division physics, Phys. Rev. ST Phys. Educ. Res. 9, 020119 (2013).

[6] O. Uhden et al., Modelling mathematical reasoning in physics education, Sci. Educ. 21, 485 (2012).

[7] B. Modir, J. D. Thompson, and E. C. Sayre, Students' epistemological framing in quantum mechanics problem solving, Phys. Rev. ST Phys. Educ. Res. 13, 020108 (2017).

[8] D. N. Chari, H. D. Nguyen, D. A. Zollman, and E. C. Sayre, Student and instructor framing in upper-division physics, Am. J. Phys. 87, 875 (2019).

[9] J. Tuminaro and E. F. Redish, Elements of a cognitive model of physics problem solving: Epistemic games, Phys. Rev. ST Phys. Educ. Res. 3, 020101 (2007).

[10] R. R. Bajracharya and J. R. Thompson, Analytical derivation: An epistemic game for solving mathematically based physics problems, Phys. Rev. ST Phys. Educ. Res. 12, 010124 (2016).

[11] R. R. Bajracharya, P. J. Emigh, and C. A. Manogue, Students' strategies for solving a multirepresentational partial derivative problem in thermodynamics, Phys. Rev. Phys. Educ. Res. 15, 020124 (2019).

[12] E. Gire and E. Price, Arrows as anchors: An analysis of the material features of electric field vector arrows, Phys. Rev. ST Phys. Educ. Res. 10, 020112 (2014).

[13] E. Gire and E. Price, Structural features of algebraic quantum notations, Phys. Rev. ST Phys. Educ. Res. 11, 020109 (2015).
[14] P. B. Kohl and N. D. Finkelstein, Student representational competence and self-assessment when solving physics problems, Phys. Rev. ST Phys. Educ. Res. 1, 010104 (2005).

[15] P. B. Kohl and N. D. Finkelstein, Effects of representation on students solving physics problems: A fine-grained characterization, Phys. Rev. ST Phys. Educ. Res. 2, 010106 (2006).

[16] R. S. Russ and T. O. B. Odden, Intertwining evidence- and model-based reasoning in physics sensemaking: An example from electrostatics, Phys. Rev. ST Phys. Educ. Res. 13, 020105 (2017).

[17] T. O. B. Odden and R. S. Russ, Defining sensemaking: Bringing clarity to a fragmented theoretical construct, Sci. Educ. 103, 187 (2018).

[18] B.W. Dreyfus et al., Mathematical sense-making in quantum mechanics: An initial peek, Phys. Rev. ST Phys. Educ. Res. 13, 020141 (2017).

[19] K. T. Hahn, Student Evaluative Sensemaking on Homework in Intermediate Mechanics, Master's thesis, Oregon State University, 2018.

[20] M. M. Hull, E. Kuo, A. Gupta, and A. Elby, Problemsolving rubrics revisited: Attending to the blending of informal conceptual and formal mathematical reasoning, Phys. Rev. ST Phys. Educ. Res. 9, 010105 (2013).

[21] E. Kuo, M. M. Hull, A. Gupta, and A. Elby, How students blend formal and conceptual mathematical reasoning in solving physics problems, Sci. Educ. 97, 32 (2013).

[22] E. Kuo et al., Assessing mathematical sensemaking in physics through calculation-concept crossover, Phys. Rev. Phys. Educ. Res. 16, 020109 (2020).

[23] https://www.lexico.com/en/definition/sense-making.

[24] L. Vygotsky, Mind in Society (Harvard University Press, Cambridge, England, 1978).

[25] G. Lakoff and R. Nunez, Where Mathematics Comes From (Basic Books, New York, 2000).

[26] M. Cole, Cultural Psychology: A Once and Future Discipline (Harvard University Press, Cambridge, England, 1998).

[27] Y. Engestrom, R. Miettinen, and R. Punamaki, Perspectives on Activity Theory (Cambridge University Press, Cambridge, England, 1999). 
[28] W. M. Roth, Cultural-historical activity theory: Vygotsky's forgotten and suppressed legacy and its implication for mathematics education, Math Educ. Res. J. 24, 87 (2012).

[29] J. Dewey, Democracy and Education: An Introduction to the Philosophy of Education (Macmillan, New York, 1916).

[30] L. C. McDermott and P. S. Shaffer, Research as a guide for curriculum development: An example from introductory electricity. Part 1: Investigation of student understanding, Am. J. Phys. 60, 994 (1992).

[31] P. S. Shaffer and L. C. McDermott, Research as a guide for curriculum development: An example from introductory electricity. Part 2: Design of instructional strategies, Am. J. Phys. 60, 1003 (1992).

[32] L. C. McDermott and The Physics Education Group at the University of Washington, Physics by Inquiry Vols. I and II (New York: John Wiley \& Sons, Inc., New York, 1996).

[33] L. C. McDermott and P. S. Shaffer, Tutorials in Introductory Physics (Prentice Hall, Upper Saddle River, NJ, 2001).

[34] https://osuper.science.oregonstate.edu/content/raisingphysics-surface.

[35] D. Hammer, Student resources for learning introductory physics, Am. J. Phys. 68, S52 (2000).
[36] A. A. diSessa, Toward an epistemology of physics, Cognit. Instr. 10, 105 (1993).

[37] N. S. Podolefsky, Analogical Scaffolding: Making Meaning in Physics through Representation and Analogy, Ph.D. thesis, University of Colorado Boulder, Boulder, Colorado, 2008.

[38] E. Mazur, Peer Instruction: A User's Manual (Prentice Hall, Upper Saddle River, NJ, 1997).

[39] J. R. Hoehn, J. D. Gifford, and N. D. Finkelstein, Epistemic stances toward group work in learning physics: Interactions between epistemology and social dynamics in a collaborative problem solving context, arXiv:2005.02425.

[40] J. D. Gifford and N.D. Finkelstein, Categorizing mathematical sense making and an example of how physics understanding can support mathematical understanding, in Proceedings of the 2019 Physical Education Research Conference, Provo, UT (AP, New York, 2019).

[41] E.F. Redish, Teaching Physics with the Physics Suite (John Wiley \& Sons Inc., New York, 2003).

[42] D. J. Griffiths, Introduction to Quantum Mechanics, 2nd ed. (Pearson Prentice Hall, Upper Saddle River, NJ, 2004).

[43] J. R. Hoehn, J. D. Gifford, and N. D. Finkelstein, Investigating the dynamics of ontological reasoning across contexts in quantum physics, Phys. Rev. Phys. Educ. Res. 15, 010124 (2019). 